\title{
The search for the magnetic precursor of C-type shocks in young molecular outflows
}

\author{
J. F. Roberts ${ }^{1}$, I. Jiménez-Serra ${ }^{2}$, A. Gusdorf ${ }^{3,4}$, and J. Martín-Pintado ${ }^{1}$ \\ ${ }^{1}$ Centro de Astrobiología (CSIC/INTA), Instituto Nacional de Técnica Aeroespacial, Ctra de Torrejón a Ajalvir, km 4, \\ 28850 Torrejón de Ardoz, Madrid, Spain \\ e-mail: robertsj@cab.inta-csic.es \\ 2 Harvard-Smithsonian Center for Astrophysics, 60 Garden St, Cambridge, MA 02138, USA \\ 3 Max-Planck-Institut für Radioastronomie, Auf dem Hügel 69, 53121 Bonn, Germany \\ ${ }^{4}$ LERMA, UMR 8112 du CNRS, Observatoire de Paris, École Normale Supérieure, 24 rue Lhomond, 75231 Paris Cedex 05, France
}

Received 12 February 2012 / Accepted 28 June 2012

\section{ABSTRACT}

\begin{abstract}
The detection of very narrow $\mathrm{SiO}$ emission and the higher degree of excitation of the ion fluid compared to the neutral one towards the protostar L1448-mm have been interpreted as signatures of the magnetic precursor of C-shocks. Since this is the only source where these features have been observed together, we carried out a survey to map the $J=2 \rightarrow 1$ and $8 \rightarrow 7$ lines of SiO, and the $J=1 \rightarrow 0$ and $4 \rightarrow 3$ lines of $\mathrm{H}^{13} \mathrm{CO}^{+}$(as the representative ion) and $\mathrm{HN}^{13} \mathrm{C}$ (as the representative neutral species) towards four class 0/I low-mass protostars known to have young outflows (L1448-IRS3, NGC 1333-IRAS 4A/B and SSV 13, VLA 1623 and IRAS 20353). As previously reported, towards L1448-IRS3 and NGC 1333 strong, narrow SiO $2 \rightarrow 1$ is detected, and we also find a velocity component towards each outflow which shows high a $\mathrm{H}^{13} \mathrm{CO}^{+} 4 \rightarrow 3 / 1 \rightarrow 0$ integrated intensity ratio compared to that of $\mathrm{HN}^{13} \mathrm{C}$. Towards VLA 1623, we find very weak, narrow $\mathrm{SiO}$, and a component that is visible in $\mathrm{H}^{13} \mathrm{CO}^{+}$but not at all in $\mathrm{HN}^{13} \mathrm{C}$. For IRAS 20353, we find neither narrow $\mathrm{SiO}$ nor high $\mathrm{H}^{13} \mathrm{CO}^{+} 4 \rightarrow 3 / 1 \rightarrow 0$ flux ratios.

Previous work explained the higher degree of excitation of $\mathrm{H}^{13} \mathrm{CO}^{+}$by the selective excitation of ions by electrons, whose abundance is expected to increase at the precursor stage. Here, however, by considering a range of kinetic temperatures for the gas, we find that it is possible to fit the observations with a single temperature and density, without an increased electron abundance, despite the large differences in $4 \rightarrow 3 / 1 \rightarrow 0$ ratio between the two molecules. The high $\mathrm{H}^{13} \mathrm{CO}^{+} 4 \rightarrow 3 / 1 \rightarrow 0$ flux ratios may simply be due to protostellar heating. However, the hypothesis that narrow $\mathrm{SiO}$ is formed at the precursor stage of shocks remains plausible, since other explanations require either the presence of shocks with larger dynamical timescales, or predict the narrow $\mathrm{SiO}$ to have a larger spatial extent than it appears to have towards the sources L1448-IRS3 and VLA 1623. Larger scale maps of the narrow SiO emission are needed to confirm that it is confined to the regions around protostars (as would be expected in the precursor scenario), and mapping of different ion and neutral species is necessary to confirm whether there are indeed precursor regions surrounding young outflows where the ion and neutral species behave as separate fluids.
\end{abstract}

Key words. stars: formation - ISM: jets and outflows - shock waves - submillimeter: ISM - infrared: ISM

\section{Introduction}

Molecular outflows are associated with the earliest stages of protostellar evolution, and play an important role in the star formation process by dispersing the surrounding natal core of the protostar, and by injecting turbulent energy into the molecular cloud. Shocks propagate at the interface between the outflow and the surrounding ambient material, dramatically modifying the density, temperature and chemistry of the unperturbed gas. In molecular dark clouds, where the fractional ionisation of the gas is very low $\left(X(\mathrm{e}) \leq 10^{-7}\right)$, when the magnetosonic speed is greater than the speed of the shock, the magnetic field is compressed ahead of the shock front, causing the charged fluid to accelerate and decouple from the neutral one (Mullan 1971; Draine 1980). The decoupled region ahead of the shock front is known as the magnetic precursor. If the magnetic field is sufficiently large, momentum transfer through collisions between the charged and neutral species in the precursor smooths out the discontinuity in the neutral fluid, resulting in a C-type (continuous) shock. The structure of a typical C-type shock is shown in Fig. 1 (reproduced from Jiménez-Serra et al. 2008). The shock structure was calculated using a parameterised model, with a shock velocity of $40 \mathrm{~km} \mathrm{~s}^{-1}$, magnetic field strength $450 \mu \mathrm{G}$, initial $\mathrm{H}_{2}$ density of $10^{5} \mathrm{~cm}^{-3}$ and an ambient gas temperature of $10 \mathrm{~K}$. In this case, since there is no longer a clear-cut shock front, we define the "precursor" as the region where the difference in velocity of the ion and neutral fluids is increasing, which roughly coincides with the region before the temperature of the neutrals reaches its maximum (Flower \& Pineau des Forêts 2003). In the example in Fig. 1 the precursor length is $0.0005-0.001 \mathrm{pc}$.

As a consequence of the precursor interaction, the velocity decoupling between the ion and neutral fluids produces the sputtering of (mostly charged) dust grains, leading to the injection of large amounts of molecular material into the gas phase (see e.g. Caselli et al. 1997; Gusdorf et al. 2008; Jiménez-Serra et al. 2008). In addition, the densities (and fractional abundances) of the ion and electron fluids are expected to be enhanced by several orders of magnitude in the precursor, due to both the compression of the charged fluid and also UV fluorescence radiation which is generated after the collisional excitation of $\mathrm{H}_{2}$ at the precursor C-shock stage (see e.g. Flower et al. 1996; Flower \& Pineau des Forêts 2003).

Observationally, there is currently evidence that points towards the detection of the signatures of the magnetic precursor of $\mathrm{C}$-shock waves towards the very young outflow emanating 


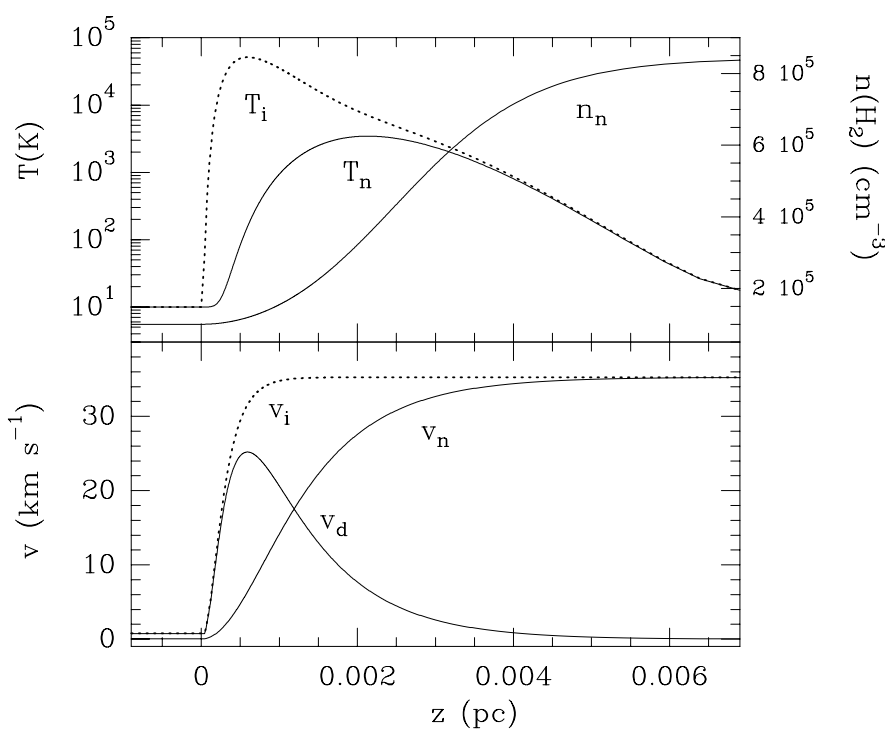

Fig. 1. Structure of a typical C-type shock from the parametric model of Jiménez-Serra et al. (2008). This model has a shock velocity of $40 \mathrm{~km} \mathrm{~s}^{-1}$, magnetic field strength of $450 \mu \mathrm{G}$, initial $\mathrm{H}_{2}$ density of $10^{5} \mathrm{~cm}^{-3}$ and temperature of $10 \mathrm{~K}$. The top panel shows the temperature of the ion and neutral fluids ( $T_{\mathrm{i}}$ and $T_{\mathrm{n}}$ respectively) and the neutral gas density $\left(n_{\mathrm{n}}\right)$. The bottom panel shows the ion and neutral fluid velocities $\left(v_{\mathrm{i}}\right.$ and $\left.v_{\mathrm{n}}\right)$ in a frame co-moving with the pre-shock gas, and $v_{\mathrm{d}}$ is the velocity difference between the two fluids.

from the L1448-mm source (dynamical age of few $1000 \mathrm{yr}$; Bachiller et al. 1990). Firstly, Jiménez-Serra et al. (2004) reported the detection of very narrow (linewidth of only $\sim 0.6 \mathrm{~km} \mathrm{~s}^{-1}$ ) $\mathrm{SiO}$ emission arising from material at almost ambient velocities (the precursor component) towards L1448-mm. This narrow feature, which clearly differs from the broad $\mathrm{SiO}$ line profiles associated with high velocity post-shock gas (typical linewidths of $\Delta v=10-50 \mathrm{~km} \mathrm{~s}^{-1}$ ) (Martín-Pintado et al. 1992), could be the result of the sputtering of grain mantles at the precursor stage (Jiménez-Serra et al. 2008, 2009). By performing a multi-transition analysis of the ion and neutral molecular emission towards L1448-mm, Jiménez-Serra et al. (2006, hereafter JS06) reported the high degree of excitation of molecular ions such as $\mathrm{H}^{13} \mathrm{CO}^{+}$with respect to neutral molecules such as $\mathrm{HN}^{13} \mathrm{C}$ (hereafter referred to as the "ion over-excitation"), in the precursor component (red-shifted by $0.5 \mathrm{~km} \mathrm{~s}^{-1}$ with respect to the ambient gas) towards this outflow. JS06 argued that the electron fractional abundance enhancement that is predicted at the precursor stage of C-shocks could cause the selective excitation of ions, because the collisional coefficients for electrons with molecular ions are larger than those with neutral species at low temperatures. For the case of $\mathrm{H}^{13} \mathrm{CO}^{+}$and $\mathrm{HN}^{13} \mathrm{C}$, at temperatures of $10-30 \mathrm{~K}$, the collisional coefficients of $\mathrm{H}^{13} \mathrm{CO}^{+}$with electrons are at least four times greater than those for $\mathrm{HN}^{13} \mathrm{C}$ with electrons for the $J=4 \rightarrow 3$ and $1 \rightarrow 0$ transitions, using the electron collisional coefficients from Faure et al. (2007) and Faure et al. (priv. comm.). More recently, Roberts et al. (2010) have shown that the ion over-excitation in the precursor component towards L1448-mm is restricted to the regions where narrow $\mathrm{SiO}$ is detected, and using observations of rarer isotopologues of $\mathrm{H}^{13} \mathrm{CO}^{+}$and $\mathrm{HN}^{13} \mathrm{C}$ they found it unlikely that it is produced by a large optical depth effect if the emission is extended. Interferometric observations have also shown that the narrow $\mathrm{SiO}$ emission is very compact (size of $\leq 0.004 \mathrm{pc}$ ) and arises from regions at the interface between the post-shock gas and the ambient medium (Jiménez-Serra et al. 2011).

All this suggests that narrow $\mathrm{SiO}$ and the over-excitation of the ion fluid are the signatures of the interactions at work exclusively in the magnetic precursor of C-shocks. As a consequence, one would expect these features to be common towards other young molecular outflows around low-mass protostars. However, L1448-mm is the only object where narrow $\mathrm{SiO}$ emission and an ion over-excitation have been reported together so far.

In this paper, we present preliminary results from a multitransition analysis of the $\mathrm{SiO}, \mathrm{H}^{13} \mathrm{CO}^{+}$and $\mathrm{HN}^{13} \mathrm{C}$ emission towards four other class $0 / \mathrm{I}$ protostars with young molecular outflows (NGC 1333-IRAS 4A/B and SSV 13, L1448-IRS3, VLA 1623 and IRAS 20353+6742 (hereafter IRAS 20353)), to see if these outflows also exhibit the features given above. We restrict our search to just low-mass protostars because in the case of high-mass objects, the strong UV field emanating from the protostar is likely to interfere in the study of the ion excitation and chemistry. For regions where we identify an $\mathrm{H}^{13} \mathrm{CO}^{+}$overexcitation, we then use a large velocity gradient (LVG) excitation model to determine the physical conditions of the gas and to explore in detail the excitation of $\mathrm{H}^{13} \mathrm{CO}^{+}$and $\mathrm{HN}^{13} \mathrm{C}$. We compare the effects of both increasing kinetic temperature and electron fractional abundance on the excitation of the two molecules, to ascertain whether or not an electron abundance enhancement is needed to explain the observed differences in excitation.

These data belong to a larger program carried out with the IRAM $30 \mathrm{~m}$ and the JCMT telescopes, to on-the-fly map this emission across the entire outflowing regions. The complete dataset will be published in detail in a forthcoming paper. The observing strategy and details of the observations are reported in Sects. 2 and 3, and the results in Sect. 4. These results are analysed in detail by using our LVG code in Sect. 5, and the conclusions are presented in Sect. 6.

\section{Observing strategy}

The molecules $\mathrm{H}^{13} \mathrm{CO}^{+}$and $\mathrm{HN}^{13} \mathrm{C}$ were chosen as the representative ion and neutral species because (i) the frequencies of their $J=1 \rightarrow 0,3 \rightarrow 2$ and $4 \rightarrow 3$ lines are such that they can be observed with few set-ups in the JCMT and IRAM $30 \mathrm{~m}$ telescopes, and most importantly; (ii) they both have similar critical densities and are likely to have reasonably similar molecular abundances in star forming molecular clouds, and hence are likely to trace the same gas. Table 1 shows the critical densities of the $J=1 \rightarrow 0$ and $4 \rightarrow 3$ transitions of $\mathrm{H}^{13} \mathrm{CO}^{+}$and $\mathrm{HN}^{13} \mathrm{C}$ at various temperatures from 10 to $100 \mathrm{~K}$, and shows that the $1 \rightarrow 0$ lines both trace gas with densities greater than a few times $10^{5} \mathrm{~cm}^{-3}$, and the $4 \rightarrow 3$ lines both trace gas with densities greater than a few times $10^{6}-10^{7} \mathrm{~cm}^{-3}$ for this temperature range. Gas-phase chemical models show that under dark cloud conditions, $\mathrm{HCO}^{+}$and $\mathrm{HNC}$ should have abundances of similar orders of magnitude (Woodall et al. 2007), so assuming that there are no ${ }^{13} \mathrm{C}$ fractionation effects, the abundances of $\mathrm{H}^{13} \mathrm{CO}^{+}$and $\mathrm{HN}^{13} \mathrm{C}$ should also be similar to each other. We note however that the $\mathrm{HN}^{(13)} \mathrm{C}$ abundance is expected to drop rapidly for temperatures above $24 \mathrm{~K}$, when it is converted into $\mathrm{H}^{(13)} \mathrm{CN}$ (Hirota et al. 1998). In addition, the critical densities of $\mathrm{HN}^{13} \mathrm{C}$ are generally about a factor of 2 higher than those of $\mathrm{H}^{13} \mathrm{CO}^{+}$for the $1 \rightarrow 0$ and $4 \rightarrow 3$ transitions, so even for similar abundances one would expect the $\mathrm{H}^{13} \mathrm{CO}^{+}$lines to be stronger and possibly more extended than those of $\mathrm{HN}^{13} \mathrm{C}$. 
Table 1. Critical $\mathrm{H}_{2}$ densities in $\mathrm{cm}^{-3}$ of the $1 \rightarrow 0$ and $4 \rightarrow 3$ transitions of $\mathrm{H}^{13} \mathrm{CO}^{+}$and $\mathrm{HN}^{13} \mathrm{C}$ at various temperatures.

\begin{tabular}{lccccc}
\hline \hline Line & $10 \mathrm{~K}$ & $30 \mathrm{~K}$ & $50 \mathrm{~K}$ & $70 \mathrm{~K}$ & $100 \mathrm{~K}$ \\
\hline $\mathrm{H}^{13} \mathrm{CO}^{+}(1 \rightarrow 0)$ & $1.5 \times 10^{5}$ & $1.8 \times 10^{5}$ & $1.9 \times 10^{5}$ & $2.0 \times 10^{5}$ & $2.1 \times 10^{5}$ \\
$\mathrm{HN}^{13} \mathrm{C}(1 \rightarrow 0)$ & $2.8 \times 10^{5}$ & $3.2 \times 10^{5}$ & $3.5 \times 10^{5}$ & $3.8 \times 10^{5}$ & $4.1 \times 10^{5}$ \\
$\mathrm{H}^{13} \mathrm{CO}^{+}(4 \rightarrow 3)$ & $8.2 \times 10^{6}$ & $8.4 \times 10^{6}$ & $8.4 \times 10^{6}$ & $8.2 \times 10^{6}$ & $7.8 \times 10^{6}$ \\
$\mathrm{HN}^{13} \mathrm{C}(4 \rightarrow 3)$ & $2.0 \times 10^{7}$ & $2.0 \times 10^{7}$ & $2.1 \times 10^{7}$ & $2.1 \times 10^{7}$ & $2.1 \times 10^{7}$ \\
\hline
\end{tabular}

Notes. The critical densities were calculated using data from the Leiden Atomic and Molecular Database (Schöier et al. 2005). For HN ${ }^{13} \mathrm{C}$ the collisional coefficients for HNC (Dumouchel et al. 2010) were used, which were calculated using He as the collisional partner, then scaled by 1.37 to represent collisions with $\mathrm{H}_{2}$. For $\mathrm{H}^{13} \mathrm{CO}^{+}$the collisional coefficients of $\mathrm{HCO}^{+}$with $\mathrm{H}_{2}$ (Flower 1999) were used.

The $\mathrm{SiO} 2 \rightarrow 1$ and $8 \rightarrow 7$ transitions were selected to probe the low- and high-excitation shocked gas possibly affected by the early interaction of the precursor of C-shocks.

\section{Observations}

The $\mathrm{SiO} J=2 \rightarrow 1, \mathrm{H}^{13} \mathrm{CO}^{+} J=1 \rightarrow 0$ and $\mathrm{HN}^{13} \mathrm{C} J=$ $1 \rightarrow 0$ lines (all at $\sim 87 \mathrm{GHz}$ ) were observed in June 2010 with the receiver EMIR (Eight MIxer Receiver) at the IRAM (Instituto de Radioastonomía Milimétrica) $30 \mathrm{~m}$ telescope, at Pico Veleta (Spain), towards the protostars NGC 1333-IRAS 4A/B and SSV 13, L1448-IRS3, VLA 1623 and IRAS 20353. The beam size at this frequency is $\sim 29^{\prime \prime}$. We used the on-the-fly mapping, position switched observing mode at a rate of $4^{\prime \prime} \mathrm{s}^{-1}$, which covered total areas of approximately 6 arcmin squared. The central coordinates of the OTF maps were $\left(\alpha_{\mathrm{J} 2000}=03^{\mathrm{h}} 29^{\mathrm{m}} 03^{\mathrm{s}} \cdot 0\right.$, $\left.\delta_{\mathrm{J} 2000}=31^{\circ} 16^{\prime} 05^{\prime \prime} \cdot 6\right)$ for NGC $1333,\left(\alpha_{\mathrm{J} 2000}=03^{\mathrm{h}} 25^{\mathrm{m}} 35^{\mathrm{s}} .9\right.$, $\left.\delta_{\mathrm{J} 2000}=30^{\circ} 45^{\prime} 17^{\prime \prime} \cdot 0\right)$ for L1448-IRS3, $\left(\alpha_{\mathrm{J} 2000}=16^{\mathrm{h}} 26^{\mathrm{m}} 26^{\mathrm{s}} .3\right.$, $\delta_{\mathrm{J} 2000}=-24^{\circ} 24^{\prime} 30^{\prime}$. 0$)$ for VLA 1623, and $\left(\alpha_{\mathrm{J} 2000}=\right.$ $20^{\mathrm{h}} 35^{\mathrm{m}} 45^{\mathrm{s}} .9, \delta_{\mathrm{J} 2000}=67^{\circ} 53^{\prime} 02^{\prime \prime}$. 0 ) for IRAS 20353.

The off reference positions with respect to the central coordinates of the maps were taken from the literature for L1448-IRS3 (JS06; (800", 0)) and NGC 1333 (Choi et al., 2004; (900", 0)). For VLA 1623 and IRAS 20353 off positions of $\left(0,-800^{\prime \prime}\right)$ and $\left(-800^{\prime \prime}, 0\right)$ were used, and were checked to be free of emission in the $1 \rightarrow 0$ lines down to an rms of $\leq 0.07 \mathrm{~K}$ at $0.1 \mathrm{~km} \mathrm{~s}^{-1}$ binning. The spectrometer ("back-end") VESPA (VErsatile SPectrometer Array) was used, providing a spectral resolution of $40 \mathrm{kHz}$. This corresponds to a velocity resolution of $\sim 0.14 \mathrm{~km} \mathrm{~s}^{-1}$ at $87 \mathrm{GHz}$. Pointing was checked on a nearby bright source every one to two hours, and the system temperatures ranged from 70 to $280 \mathrm{~K}$.

The SiO $J=8 \rightarrow 7, \mathrm{H}^{13} \mathrm{CO}^{+} J=4 \rightarrow 3$ and $\mathrm{HN}^{13} \mathrm{C} J=$ $4 \rightarrow 3$ lines at $347-348 \mathrm{GHz}$ were observed towards the same outflows using the HARP-B (Heterodyne ARray Program-B) receiver at the James Clerk Maxwell Telescope (JCMT) at Mauna Kea (Hawaii), in September 2010. We mapped this emission by using the HARP5 map-centred jiggle-map mode in position switching. The off positions used in the observations are the same as those above. The ACSIS (Auto-Correlation Spectrometer Imaging System) spectrometer provided resolutions of $61 \mathrm{kHz}$ for $\mathrm{H}^{13} \mathrm{CO}^{+} 4 \rightarrow 3$ and $\mathrm{HN}^{13} \mathrm{C} 4 \rightarrow 3$, and $31 \mathrm{kHz}$ for $\mathrm{SiO} 8 \rightarrow 7$, corresponding to velocity resolutions of $0.05 \mathrm{~km} \mathrm{~s}^{-1}$ and $0.03 \mathrm{~km} \mathrm{~s}^{-1}$ at $347 \mathrm{GHz}$, respectively. The beam size of the observations was $14^{\prime \prime}$. Pointing was checked every one to two hours on a nearby bright source, and system temperatures ranged from 230 to $395 \mathrm{~K}$.

All the intensities were calibrated in units of antenna temperature, and for the LVG modelling we convert to $T_{\mathrm{MB}}$ using beam efficiencies of 0.82 and 0.63 for the $\sim 90$ and $\sim 350 \mathrm{GHz}$ lines respectively.

\section{Results}

Maps of the $\mathrm{SiO} 2 \rightarrow 1, \mathrm{H}^{13} \mathrm{CO}^{+} 1 \rightarrow 0, \mathrm{HN}^{13} \mathrm{C} 1 \rightarrow 0$, and $\mathrm{H}^{13} \mathrm{CO}^{+} 4 \rightarrow 3$ integrated intensities are shown in Figs. 2 to 5 for each source. The emission was integrated over $5.5-9.5 \mathrm{~km} \mathrm{~s}^{-1}$ for NGC 1333, 0.5-7 $\mathrm{km} \mathrm{s}^{-1}$ for L1448-IRS3, 2.5-4.6 $\mathrm{km} \mathrm{s}^{-1}$ for VLA 1623, and $2-3.3 \mathrm{~km} \mathrm{~s}^{-1}$ for IRAS 20353. These velocity ranges were chosen such that they include all the narrow $\mathrm{SiO}$, $\mathrm{H}^{13} \mathrm{CO}^{+}$and $\mathrm{HN}^{13} \mathrm{C}$ emission. We do not show the $\mathrm{HN}^{13} \mathrm{C} 4 \rightarrow 3$ integrated intensities because it was not detected towards any of the sources above the $3 \sigma$ limit.

We also plot the $\mathrm{H}^{13} \mathrm{CO}^{+} 4 \rightarrow 3 / 1 \rightarrow 0$ integrated intensity ratio maps, which were calculated by regridding the $\mathrm{H}^{13} \mathrm{CO}^{+}$ $4 \rightarrow 3$ maps onto the same grid as the $\mathrm{H}^{13} \mathrm{CO}^{+} 1 \rightarrow 0$ maps using the task "reproject" from the CLASS analysis software included in the GILDAS package ${ }^{1}$, and then using the task "combine" to calculate their ratio. Pixels with an integrated intensity of less than $3 \sigma$ (where $\sigma$ is the map rms noise) in either molecule were considered invalid (apart from for NGC 1333 where we allow pixels with an $\mathrm{H}^{13} \mathrm{CO}^{+} 4 \rightarrow 3$ integrated intensity greater than $1 \sigma$ to be used, because of the small spatial extent of the emission from this line). For IRAS 20353, even when using a threshold of $1 \sigma$ for the $4 \rightarrow 3$ line there are insufficient valid pixels, therefore we do not show this map.

In addition, we also plot the Spitzer IRAC channel $2(4.5 \mu \mathrm{m})$ map to put the observed regions into context. The $4.5 \mu \mathrm{m}$ emission reveals any knots of shocked $\mathrm{H}_{2}$ gas (for example, south of L1448-IRS3, and towards the Herbig-Haro objects south-east of SSV 13 in the NGC 1333 region), and also reflection nebulae (for example, the strong emission north-east of VLA 1623, Dent et al. 1995) towards the sources. The locations of nearby protostars are marked with squares and Herbig-Haro objects with triangles, and the location of the offsets selected to study in more detail (see below) are marked by a cross on each map.

The maps of the $\mathrm{H}^{13} \mathrm{CO}^{+} 1 \rightarrow 0$ and $\mathrm{HN}^{13} \mathrm{C} 1 \rightarrow 0$ lines show that, in general, the spatial distribution of these two molecules are similar, adding confidence to our assumption that they trace the same gas. The $\mathrm{H}^{13} \mathrm{CO}^{+} 1 \rightarrow 0$ emission tends to be more widespread, owing to its lower critical density.

For each outflow, maps of the $\mathrm{SiO} 2 \rightarrow 1$ integrated intensity and $\mathrm{H}^{13} \mathrm{CO}^{+} 4 \rightarrow 3 / 1 \rightarrow 0$ integrated intensity ratio were inspected to identify any offsets which showed both narrow $\mathrm{SiO}$ emission and any velocity components which have very high $4 \rightarrow 3 / 1 \rightarrow 0$ integrated intensity ratios for $\mathrm{H}^{13} \mathrm{CO}^{+}$compared to the upper limit for $\mathrm{HN}^{13} \mathrm{C}$, as these may indicate the presence of the precursor (Jiménez-Serra et al. 2006). We selected a single offset from each outflow which best satisfied these criteria. The spectra from the selected offsets, smoothed to a velocity resolution of $0.1-0.15 \mathrm{~km} \mathrm{~s}^{-1}$, are shown in Fig. 6, and the observed parameters are given in Table 2. To take into account the different

${ }^{1}$ http://wWw.iram.fr/IRAMFR/GILDAS 

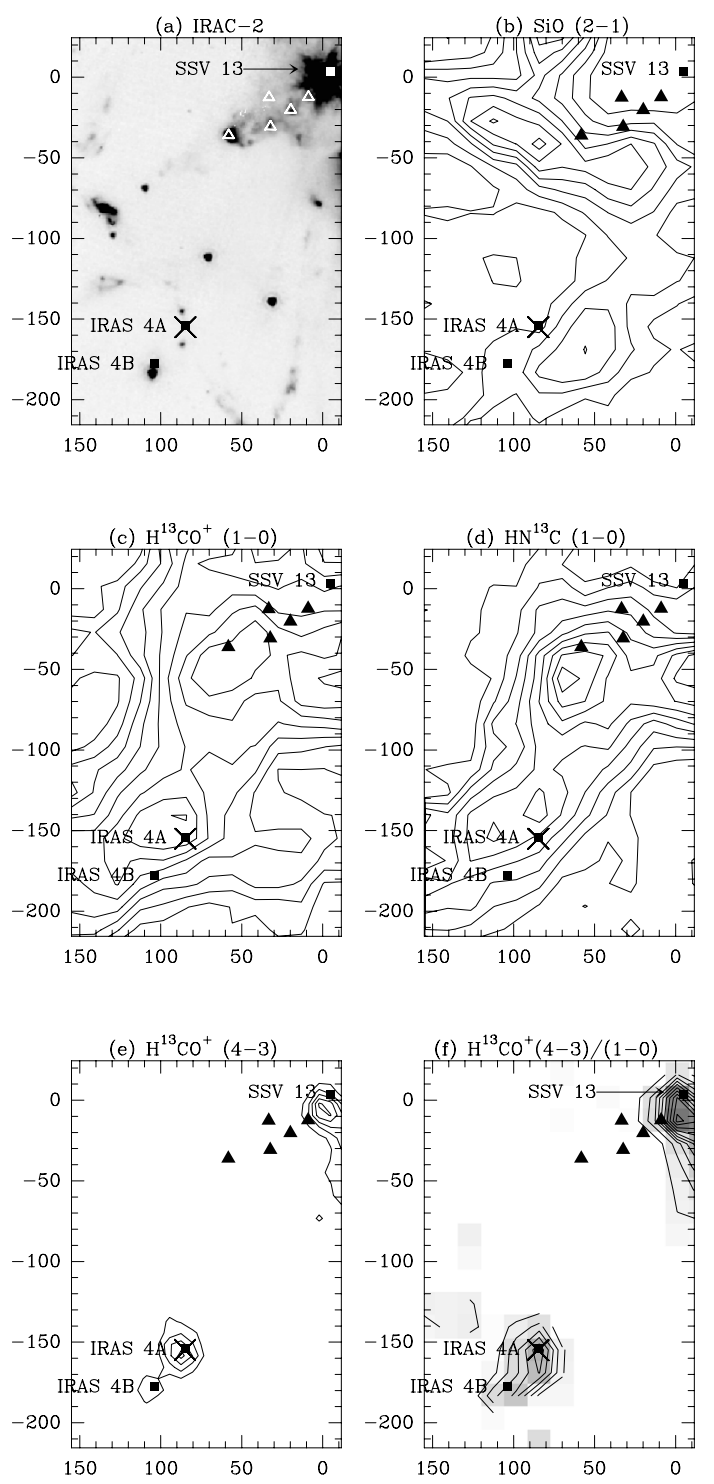

Fig. 2. Maps of the NGC 1333 region. Offsets are relative to the positions given in Sect. 3. a) Shows the Spitzer IRAC channel 2 emission ( $4.5 \mu \mathrm{m}$, retrieved from the Spitzer archive), and b) to e) show the integrated intensity maps of $\mathrm{SiO} 2 \rightarrow 1, \mathrm{H}^{13} \mathrm{CO}^{+} 1 \rightarrow 0, \mathrm{HN}^{13} \mathrm{C} 1 \rightarrow 0$, and $\mathrm{H}^{13} \mathrm{CO}^{+} 4 \rightarrow 3$. For maps b) to e) the contour levels start at $5 \sigma$, where $\sigma$ is the estimated rms noise towards the centre of the map (it is higher towards the map edges). The values of $\sigma$ (and contour step) in $\mathrm{K} \mathrm{km} \mathrm{s}^{-1}$ for these maps are: b) $0.044(0.22)$, c) $0.094(0.18)$, d) $0.056(0.10)$, and e) $0.052(0.26)$. Map f) shows the $\mathrm{H}^{13} \mathrm{CO}^{+} 4 \rightarrow 3 / 1 \rightarrow 0$ integrated intensity ratio, with a lowest contour level of 0.1 and step 0.05 . The greyscale image is shown to make the map easier to read. The squares show the location of nearby protostars and the triangles show the Herbig-Haro $7-11$ objects. The cross shows the location where we have extracted the single spectra.

beam sizes of the observations, these spectra have been averaged by assuming a Gaussian taper, whose FWHM corresponds to the largest beam size of the observations $\left(\sim 29^{\prime \prime}\right)$.

Note that towards every outflow, Fig. 6 shows that at least two "ambient" velocity components are detected. Despite the fact that $\mathrm{C}$-shock models predict that the ion and neutral velocities are continuous functions of time and space, Jiménez-Serra et al. (2009) showed that at early times the emission of $\mathrm{H}^{13} \mathrm{CO}^{+}$ and $\mathrm{HN}^{13} \mathrm{C}$ peak at two distinct velocity components as a consequence of the initial acceleration of ions by the precursor. The separate velocity components could have also formed by other
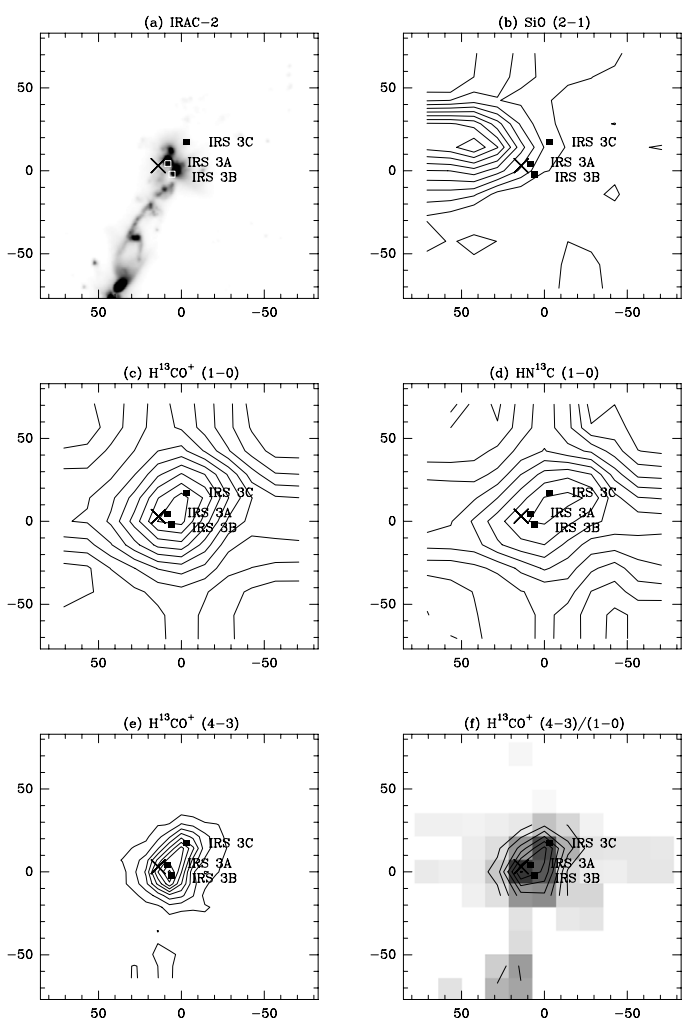

Fig. 3. As in Fig. 2, but for L1448-IRS3. The values of $\sigma$ (and contour step) in $\mathrm{K} \mathrm{km} \mathrm{s}^{-1}$ for maps b) to e) are: b) $0.016(0.081)$, c) $0.013(0.26)$, d) $0.028(0.14)$, and e) $0.030(0.15)$. The first contour level and step of the $\mathrm{H}^{13} \mathrm{CO}^{+} 4 \rightarrow 3 / 1 \rightarrow 0$ integrated intensity ratio map f) are 0.08 and 0.04 , respectively.
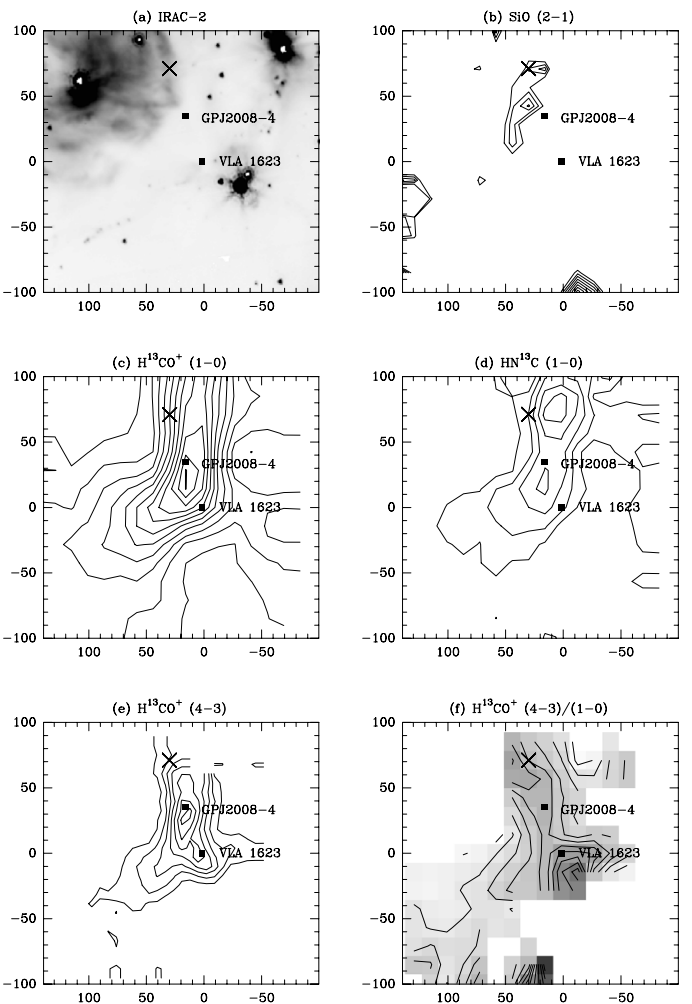

Fig. 4. As in Fig. 2, but for VLA 1623. The values of $\sigma$ (and contour step) in $\mathrm{K} \mathrm{km} \mathrm{s}^{-1}$ for maps b) to e) are: b) $0.025(0.027)$, c) $0.057(0.54)$, d) $0.030(0.15)$, and e) $0.065(0.33)$. The first contour level and step of the $\mathrm{H}^{13} \mathrm{CO}^{+} 4 \rightarrow 3 / 1 \rightarrow 0$ integrated intensity ratio map f) are 0.01 and 0.05 , respectively. 
Table 2. Observed parameters of the $\mathrm{H}^{13} \mathrm{CO}^{+}, \mathrm{HN}^{13} \mathrm{C}$ and $\mathrm{SiO}$ lines.

\begin{tabular}{|c|c|c|c|c|c|c|c|c|c|c|c|c|}
\hline \multirow[b]{2}{*}{ Line } & \multicolumn{3}{|c|}{ NGC 1333} & \multicolumn{3}{|c|}{ L1448-IRS3 } & \multicolumn{3}{|c|}{ VLA 1623} & \multicolumn{3}{|c|}{ IRAS 20353} \\
\hline & $\begin{array}{c}v_{\mathrm{lsr}} \\
\left(\mathrm{km} \mathrm{s}^{-1}\right)\end{array}$ & $\begin{array}{c}\Delta v \\
\left(\mathrm{~km} \mathrm{~s}^{-1}\right)\end{array}$ & $\begin{array}{l}T_{\mathrm{A}}^{*} \\
(\mathrm{~K})\end{array}$ & $\begin{array}{c}v_{\mathrm{lsr}} \\
\left(\mathrm{km} \mathrm{s}^{-1}\right)\end{array}$ & $\begin{array}{c}\Delta v \\
\left(\mathrm{~km} \mathrm{~s}^{-1}\right)\end{array}$ & $\begin{array}{l}T_{\mathrm{A}}^{*} \\
(\mathrm{~K})\end{array}$ & $\begin{array}{c}v_{\mathrm{lsr}} \\
\left(\mathrm{km} \mathrm{s}^{-1}\right)\end{array}$ & $\begin{array}{c}\Delta v \\
\left(\mathrm{~km} \mathrm{~s}^{-1}\right)\end{array}$ & $\begin{array}{l}T_{\mathrm{A}}^{*} \\
(\mathrm{~K})\end{array}$ & $\begin{array}{c}v_{\text {lsr }} \\
\left(\mathrm{km} \mathrm{s}^{-1}\right)\end{array}$ & $\begin{array}{c}\Delta v \\
\left(\mathrm{~km} \mathrm{~s}^{-1}\right)\end{array}$ & $\begin{array}{l}T_{\mathrm{A}}^{*} \\
(\mathrm{~K})\end{array}$ \\
\hline \multirow[t]{2}{*}{$\mathrm{H}^{13} \mathrm{CO}^{+}(1 \rightarrow 0)$} & $6.94(2)$ & $0.94(4)$ & $0.696(9)$ & $4.1090(5)$ & $0.805(9)$ & $0.91(2)$ & $2.57(2)$ & $0.60(5)$ & $0.60(5)$ & $2.637(4)$ & $0.56(1)$ & $0.97(1)$ \\
\hline & $7.80(1)$ & $0.71(2)$ & $1.022(9)$ & $\begin{array}{l}4.641(2) \\
5.231(4)\end{array}$ & $\begin{array}{l}0.736(6) \\
0.746(9)\end{array}$ & $\begin{array}{l}2.00(2) \\
0.72(2)\end{array}$ & $3.467(5)$ & $0.74(1)$ & 2.61(5) & $3.00(3)$ & $0.54(9)$ & $0.11(1)$ \\
\hline \multirow[t]{2}{*}{$\mathrm{H}^{13} \mathrm{CO}^{+}(4 \rightarrow 3)$} & $6.91(2)$ & $0.84(7)$ & $0.29(1)$ & $3.97(4)$ & $1.00(8)$ & $0.14(2)$ & $2.6(1)$ & $0.9(4)$ & $0.17(5)$ & $2.62(5)$ & $0.4(1)$ & $0.051(4)$ \\
\hline & 7.81(9) & $0.6(2)$ & $0.06(1)$ & $\begin{array}{c}4.73(2) \\
5.437(7)\end{array}$ & $\begin{array}{l}0.75(3) \\
0.72(6)\end{array}$ & $\begin{array}{l}0.39(2) \\
0.20(2)\end{array}$ & $3.35(2)$ & $1.1(2)$ & $0.47(5)$ & $\sim 3.1$ & $\ldots$ & $\leq 0.027$ \\
\hline \multirow[t]{2}{*}{$\mathrm{HN}^{13} \mathrm{C}(1 \rightarrow 0)$} & $6.87(3)$ & $0.84(7)$ & $0.24(1)$ & $4.0(1)$ & $0.5(1)$ & $0.16(1)$ & $\sim 2.6$ & . & $\leq 0.044$ & $2.66(2)$ & $0.49(3)$ & $0.46(1)$ \\
\hline & $7.90(2)$ & $0.87(4)$ & $0.38(1)$ & $\begin{array}{l}4.7(1) \\
5.5(1)\end{array}$ & $\begin{array}{l}0.9(1) \\
0.7(1)\end{array}$ & $\begin{array}{l}0.97(1) \\
0.20(1)\end{array}$ & $3.58(2)$ & $0.71(6)$ & $0.42(4)$ & $3.1(1)$ & $0.7(2)$ & $0.13(2)$ \\
\hline $\mathrm{HN}^{13} \mathrm{C}(4 \rightarrow 3)$ & $\begin{array}{l}\sim 6.9 \\
\sim 7.8\end{array}$ & $\begin{array}{l}\cdots \\
\ldots\end{array}$ & $\begin{array}{l}\leq 0.032 \\
\leq 0.032\end{array}$ & $\begin{array}{l}\sim 4.0 \\
\sim 4.7\end{array}$ & $\begin{array}{l}\ldots \\
\ldots\end{array}$ & $\begin{array}{l}\leq 0.032 \\
\leq 0.032\end{array}$ & $\begin{array}{l}\sim 2.6 \\
\sim 3.5\end{array}$ & $\begin{array}{l}\cdots \\
\ldots\end{array}$ & $\begin{array}{l}\leq 0.13 \\
\leq 0.13\end{array}$ & $\begin{array}{l}\sim 2.6 \\
\sim 3.1\end{array}$ & $\begin{array}{l}\ldots \\
\ldots\end{array}$ & $\begin{array}{l}\leq 0.040 \\
\leq 0.040\end{array}$ \\
\hline \multirow[t]{2}{*}{$\mathrm{SiO}(2 \rightarrow 1)$} & $7.1(2)$ & $3.4(4)$ & $0.10(2)$ & $\begin{array}{l}\sim 5.4 \\
\sim 4.0\end{array}$ & $\begin{array}{l}\ldots \\
\ldots\end{array}$ & $\begin{array}{l}\leq 0.032 \\
\leq 0.019\end{array}$ & $2.9(1)$ & $1.3(3)$ & $0.08(2)$ & $\sim 2.6$ & $\ldots$ & $\leq 0.034$ \\
\hline & $7.83(1)$ & $0.63(4)$ & $0.31(2)$ & $\begin{array}{c}4.545(4) \\
\sim 5.4\end{array}$ & $\begin{array}{c}0.499(9) \\
\ldots\end{array}$ & $\begin{array}{l}0.36(1) \\
\leq 0.019\end{array}$ & $\ldots$ & $\ldots$ & $\ldots$ & $\sim 3.1$ & $\ldots$ & $\leq 0.034$ \\
\hline $\mathrm{SiO}(8 \rightarrow 7)$ & $\begin{array}{l}\sim 6.9 \\
\sim 7.8\end{array}$ & $\begin{array}{l}\cdots \\
\ldots\end{array}$ & $\begin{array}{l}\leq 0.031 \\
\leq 0.031\end{array}$ & $\begin{array}{l}\sim 4.0 \\
\sim 4.7 \\
\sim 5.4\end{array}$ & $\begin{array}{l}\ldots \\
\ldots \\
\ldots\end{array}$ & $\begin{array}{l}\leq 0.033 \\
\leq 0.033 \\
\leq 0.033\end{array}$ & & & & $\begin{array}{l}\sim 2.6 \\
\sim 3.1\end{array}$ & $\begin{array}{l}\cdots \\
\ldots\end{array}$ & $\begin{array}{l}\leq 0.049 \\
\leq 0.049\end{array}$ \\
\hline
\end{tabular}

Notes. For the peak intensity, $T_{\mathrm{A}}^{*}$, the number in brackets shows the estimated error on the last digit, estimated by the $1 \sigma$ noise level. For the position, $v_{\mathrm{lsr}}$, and linewidth, $\Delta v$, the errors on the last digit quoted are the values given by the fitting routine "minimize" in the CLASS analysis software, which may underestimate the true errors as they do not take into account, for example, uncertainties in the inputs to the fitting routine. The upper limits of the line intensities correspond to the $3 \sigma$ noise level in the spectra when smoothed to a velocity resolution of $\sim 0.2 \mathrm{~km} \mathrm{~s}^{-1}$. At this velocity resolution there should be 3-4 channels contained within the line, which should be sufficient to estimate the peak intensity.
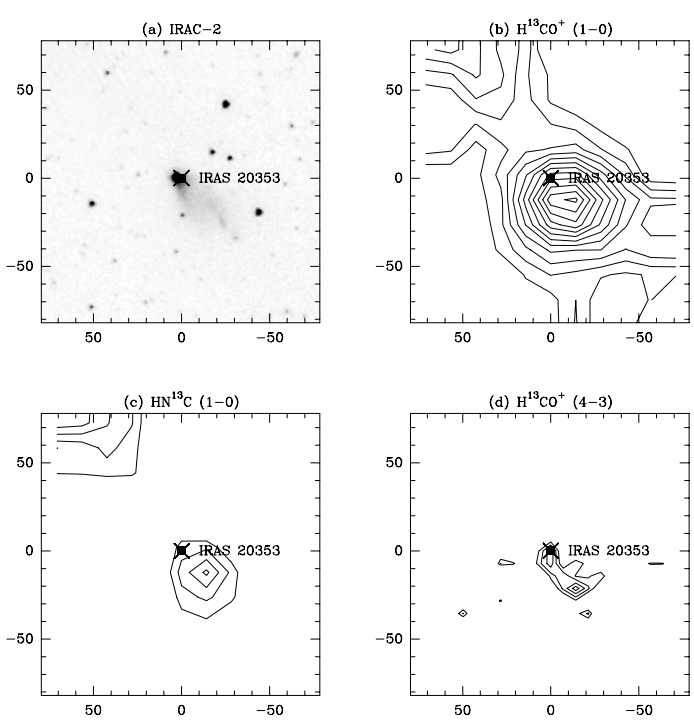

Fig. 5. As in Fig. 2, but for IRAS 20353. For this source no $\mathrm{SiO} 2 \rightarrow 1$ was detected, and the $\mathrm{H}^{13} \mathrm{CO}^{+} 4 \rightarrow 3 / 1 \rightarrow 0$ integrated intensity ratio map did not contain a sufficient number of valid pixels, therefore these maps are not shown. For maps b) to d) (showing the integrated intensities of only $\mathrm{H}^{13} \mathrm{CO}^{+} 1 \rightarrow 0, \mathrm{HN}^{13} \mathrm{C} 1 \rightarrow 0$ and $\mathrm{H}^{13} \mathrm{CO}^{+} 4 \rightarrow 3$ ), the values of $\sigma$ (and contour step) in $\mathrm{K} \mathrm{km} \mathrm{s}^{-1}$ are: b) 0.034 (0.074), c) 0.067 (0.067), and d) 0.021 (0.014). Here the first contour level of $\mathrm{H}^{13} \mathrm{CO}^{+} 4 \rightarrow 3$ (map d)) is $3 \sigma$, as opposed to $5 \sigma$ used in all the previous maps.

dynamical processes. For example Langer et al. (1996) suggested that the $8 \mathrm{~km} \mathrm{~s}^{-1}$ structure observed towards NGC 1333 is a dense shell of gas, compressed by the outflows in the region.

In Table 3, we present the $J=4 \rightarrow 3 / 1 \rightarrow 0$ integrated intensity ratios derived for every velocity component and molecule reported in Table 2 and Fig. 6. Below we discuss the results from
Table 3. $J=4 \rightarrow 3 / 1 \rightarrow 0$ integrated intensity ratios for $\mathrm{H}^{13} \mathrm{CO}^{+}$and $\mathrm{HN}^{13} \mathrm{C}$ for each component detected towards each outflow (see Fig. 6).

\begin{tabular}{lcccc}
\hline \hline & & \multicolumn{3}{c}{$4 \rightarrow 3 / 1 \rightarrow 0$ ratio } \\
Outflow & Molecule & $\mathrm{C}^{a}$ & $\mathrm{C} 2$ & $\mathrm{C} 3$ \\
\hline NGC 1333 & $\mathrm{H}^{13} \mathrm{CO}^{+}$ & 0.48 & 0.07 & $\ldots$ \\
& $\mathrm{HN}^{13} \mathrm{C}$ & $\leq 0.09$ & $\leq 0.04$ & $\ldots$ \\
L1448-IRS3 & $\mathrm{H}^{13} \mathrm{CO}^{+}$ & 0.23 & 0.26 & 0.34 \\
& $\mathrm{HN}^{13} \mathrm{C}$ & $\leq 0.29$ & $\leq 0.03$ & $\leq 0.18$ \\
VLA 1623 & $\mathrm{H}^{13} \mathrm{CO}^{+}$ & 0.59 & 0.34 & $\ldots$ \\
& $\mathrm{HN}^{13} \mathrm{C}$ & - & $\leq 0.20$ & $\ldots$ \\
IRAS 20353 & $\mathrm{H}^{13} \mathrm{CO}^{+}$ & 0.05 & $\leq 0.23$ & $\ldots$ \\
& $\mathrm{HN}^{13} \mathrm{C}$ & $\leq 0.07$ & $\leq 0.17$ & $\ldots$ \\
\hline
\end{tabular}

Notes. The integrated intensities have been converted to the $T_{\mathrm{MB}}$ scale using the beam efficiencies given in Sect. 3. ${ }^{(a)}$ The approximate central velocities (in $\mathrm{km} \mathrm{s}^{-1}$ ) of each component for each outflow are 6.9 (C1) and $7.8(\mathrm{C} 2)$ for NGC 1333, $4.0(\mathrm{C} 1), 4.7(\mathrm{C} 2)$ and $5.4(\mathrm{C} 3)$ for L1448-IRS3, 2.6 (C1) and 3.5 (C2) for VLA 1623, and 2.6 (C1) and 3.1 (C2) for IRAS 20353.

the maps, and the individual spectra extracted from the positions which best satisfied our criteria for each outflow.

\subsection{NGC 1333}

NGC 1333 is a region of intense star formation located within the Perseus molecular cloud. Its distance is estimated to be between 220 (Cernis 1990) and 350 pc (Herbig \& Jones 1983), and the complex has an ambient velocity of $\sim 6-9 \mathrm{~km} \mathrm{~s}^{-1}$. The region we have observed encompasses the IRAS $4 \mathrm{~A} / \mathrm{B}$ class 0 protostars, as well as the SSV 13 ridge (IRAS 3) which contains at least one class 0 protostar (Bachiller et al. 1998; Sandell \& Knee 2001). These protostars are all low-mass, with a total bolometric luminosity for the IRAS 4 system of $28 L_{\odot}$ (Sandell et al. 1991), 


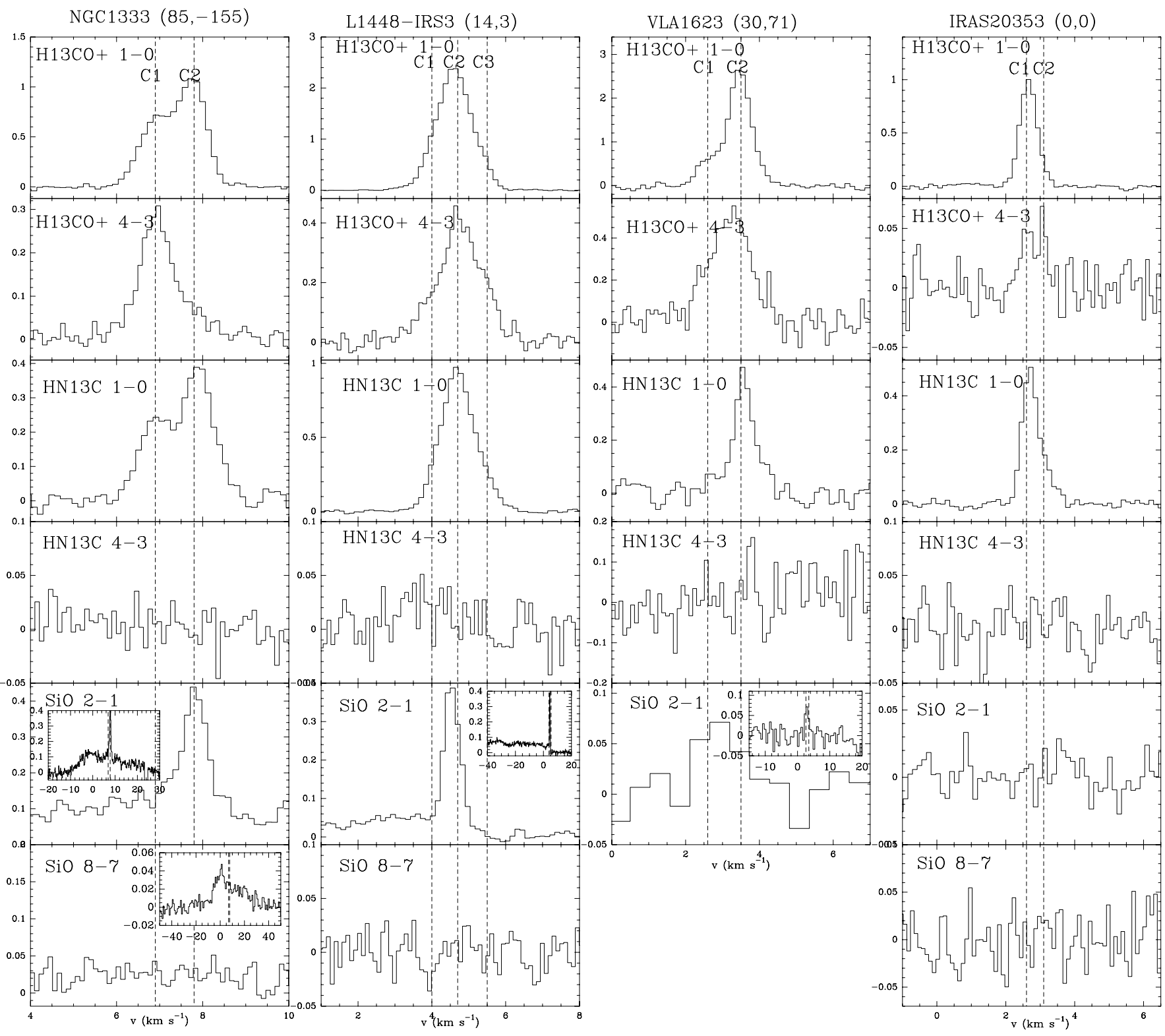

Fig. 6. Spectra of the $J=1 \rightarrow 0$ and $4 \rightarrow 3$ lines of $\mathrm{H}^{13} \mathrm{CO}^{+}$and $\mathrm{HN}^{13} \mathrm{C}$, and the $J=2 \rightarrow 1$ and $8 \rightarrow 7$ lines of SiO towards the sources NGC 1333 , L1448-IRS3, VLA 1623 and IRAS 20353, for the positions selected in Sect. 4 (given at the top of each column). To account for the different beam sizes, the spectra have been averaged by assuming a Gaussian taper, whose FWHM corresponds to the largest beam size of the observations $\left(\sim 29^{\prime \prime}\right)$. The vertical axis is line intensity in Kelvin on the $T_{\mathrm{A}}^{*}$ scale. The spectra have been smoothed to a velocity resolution of $\sim 0.1 \mathrm{~km} \mathrm{~s}{ }^{-1}$, apart from $\mathrm{SiO} 2 \rightarrow 1$ towards VLA 1623 , which has been smoothed to $\sim 0.5 \mathrm{~km} \mathrm{~s}^{-1}$ in order to see the line more clearly. The insets where plotted show the full width of the spectrum.

and 69-90 $L_{\odot}$ for the SSV 13 system (Sandell et al. 1990). Shocked gas traced by high velocity $\mathrm{SiO}$ emission indicates that the outflow emanating from IRAS 4 has a very young dynamical age of $3500 \mathrm{yr}$, and another outflow lobe, possibly generated by SSV 13, has a dynamical age of $11000 \mathrm{yr}$ (Lefloch et al. 1998b). These outflows have shaped the surrounding gas, forming a complicated structure of cavities and dense layers (Lefloch et al. 1998a).

Our observations (Fig. 2) show that, as reported by Lefloch et al. (1998b), the narrow SiO emission spreads around SSV 13 and the HH7-11 objects, and to the west of the IRAS 4A and $4 \mathrm{~B}$ cores. The $\mathrm{SiO}$ emission towards the IRAS 4 cores is slightly narrower $\left(\Delta v \sim 0.5 \mathrm{~km} \mathrm{~s}^{-1}\right)$ than the emission surrounding SSV $13\left(\Delta v \sim 1.5 \mathrm{~km} \mathrm{~s}^{-1}\right)$. The $\mathrm{H}^{13} \mathrm{CO}^{+} 4 \rightarrow 3$ emission and $\mathrm{H}^{13} \mathrm{CO}^{+} 4 \rightarrow 3 / 1 \rightarrow 0$ ratio have two peaks: one close to SSV 13 which is elongated towards the south, and another close to the IRAS $4 \mathrm{~A}$ protostar. The regions where the $\mathrm{H}^{13} \mathrm{CO}^{+} 4 \rightarrow 3 / 1 \rightarrow 0$ ratio is high do not follow the narrow $\mathrm{SiO}$ emission, which would not be expected if both these features were produced in the magnetic precursor. We selected the offset $\left(85^{\prime \prime},-155^{\prime \prime}\right)$, close to IRAS 4 A, to study in more detail rather than the emission close to SSV 13, because it has both strong, narrow $\mathrm{SiO}$ emission and a high $\mathrm{H}^{13} \mathrm{CO}^{+} J=4 \rightarrow 3 / J=$ $1 \rightarrow 0$ ratio, whereas the emission towards SSV 13 has little or no narrow $\mathrm{SiO}$ emission.

Towards $\left(85^{\prime \prime},-155^{\prime \prime}\right)$, two components are clearly visible, at $\sim 6.9$ (referred to as $\mathrm{C} 1$ in Table 3 ) and $\sim 7.8 \mathrm{~km} \mathrm{~s}^{-1}$ (C2 in Table 3). Langer et al. (1996) also observed components of emission in CS at similar velocities, and suggested that the $\sim 8 \mathrm{~km} \mathrm{~s}^{-1}$ layer is a dense compressed shell surrounding a cavity created by 
the outflows. For the $\mathrm{C} 1$ component, we find that $\mathrm{H}^{13} \mathrm{CO}^{+}$has a $4 \rightarrow 3 / 1 \rightarrow 0$ ratio 5 times greater than that for $\mathrm{HN}^{13} \mathrm{C}$. This is in contrast to component $\mathrm{C} 2$, where the upper limit of the $\mathrm{HN}^{13} \mathrm{C}$ $4 \rightarrow 3 / 1 \rightarrow 0$ ratio is similar to that of $\mathrm{H}^{13} \mathrm{CO}^{+} . \mathrm{C} 1$ therefore appears to show an over-excitation of the ion fluid similar to that reported towards L1448-mm (JS06).

Like towards L1448-mm, narrow $\left(\sim 0.6 \mathrm{~km} \mathrm{~s}^{-1}\right) \mathrm{SiO}$ is also detected towards NGC 1333. However, this narrow $\mathrm{SiO}$ emission peaks at the $\mathrm{C} 2$ velocity component where no over-excitation of $\mathrm{H}^{13} \mathrm{CO}^{+}$is reported. This behaviour differs from that observed towards L1448-mm, because the narrow $\mathrm{SiO}$ lines towards this outflow were detected at the velocity component which showed an over-excitation of the ion fluid.

Narrow $\mathrm{SiO} 8 \rightarrow 7$ was not detected, although the full spectrum (inset in Fig. 6) reveals a broad velocity wing with a terminal velocity of $\sim 40 \mathrm{~km} \mathrm{~s}^{-1}$, most likely associated with the post-shock gas.

\subsection{L1448-IRS 3}

L1448-IRS3 is a class 0/I protostellar complex (Ciardi et al. 2003), also in Perseus, lying approximately $70^{\prime \prime}$ north west of the class 0 protostar L1448- $\mathrm{mm}$. The total bolometric luminosity of the complex is $10 L_{\odot}$ (Bontemps et al. 1996), and the ambient velocity is $\sim 4-4.7 \mathrm{~km} \mathrm{~s}^{-1}$. Bachiller et al. (1990) found that L1448-mm drives a highly collimated CO outflow, the northern lobe of which appears to impact with L1448-IRS3. In addition they found that L1448-IRS3 also appears to drive its own weaker outflow, with a dynamical age of $\sim 2600 \mathrm{yr}$.

Figure 3 shows that the narrow $\mathrm{SiO}$ lies to the east of the L1448-IRS 3 complex. The emission close to L1448-IRS 3 is the narrowest $\left(\Delta v \sim 0.5 \mathrm{~km} \mathrm{~s}^{-1}\right)$, but going further east the width increases to $\Delta v \sim 2 \mathrm{~km} \mathrm{~s}^{-1}$. The $\mathrm{H}^{13} \mathrm{CO}^{+} 4 \rightarrow 3$ emission and $\mathrm{H}^{13} \mathrm{CO}^{+} 4 \rightarrow 3 / 1 \rightarrow 0$ ratio peak around the IRS3 complex, and could simply be a result of the high gas density of the protostars, but towards the east of the IRS 3 complex, where the narrow $\mathrm{SiO}$ emission is very strong, it is possible that the magnetic precursor is also contributing to the excitation of $\mathrm{H}^{13} \mathrm{CO}^{+}$. We therefore select the offset $\left(14^{\prime \prime}, 3^{\prime \prime}\right)$ to study in detail because this position has the narrowest $\mathrm{SiO}$ emission, and also a very high $\mathrm{H}^{13} \mathrm{CO}^{+} 4 \rightarrow 3 / 1 \rightarrow 0$ ratio. We note that, as for NGC 1333 , the areas with high $\mathrm{H}^{13} \mathrm{CO}^{+} 4 \rightarrow 3 / 1 \rightarrow 0$ ratio do not follow the narrow $\mathrm{SiO}$ emission.

Towards this offset, The $\mathrm{H}^{13} \mathrm{CO}^{+}$and $\mathrm{HN}^{13} \mathrm{C}$ spectra, shown in Fig. 6, are best fit using three Gaussian components, centred at velocities $\sim 4.0(\mathrm{C} 1$ in Table 3$), \sim 4.7(\mathrm{C} 2)$ and $\sim 5.5(\mathrm{C} 3) \mathrm{km} \mathrm{s}^{-1}$. The $4 \rightarrow 3 / 1 \rightarrow 0$ integrated intensity ratios (Table 3 ) show that although component $\mathrm{C} 3$ has the largest $4 \rightarrow 3 / 1 \rightarrow 0$ integrated intensity ratio, the largest difference between the $\mathrm{H}^{13} \mathrm{CO}^{+}$and $\mathrm{HN}^{13} \mathrm{C}$ ratios is seen in $\mathrm{C} 2$. For this component the $\mathrm{H}^{13} \mathrm{CO}^{+}$ $4 \rightarrow 3 / 1 \rightarrow 0$ ratio is $\gtrsim 10$ times greater than that for $\mathrm{HN}^{13} \mathrm{C}$, similar to that found towards L1448-mm, again indicating an ion over-excitation towards this component. Narrow $\mathrm{SiO} 2 \rightarrow 1$ is also detected at the velocity of $\mathrm{C} 2$, although narrow $\mathrm{SiO} 8 \rightarrow 7$ is not, implying that the excitation of narrow $\mathrm{SiO}$ gas towards this source is relatively low.

\subsection{VLA 1623}

VLA 1623 is a class 0 protostar in the $\rho$-Ophiuchus region, at a distance of $160 \mathrm{pc}$ and with a bolometric luminosity of $1 L_{\odot}$ (Bontemps et al. 1996). The ambient velocity is $3-3.5 \mathrm{~km} \mathrm{~s}^{-1}$. It drives a highly collimated and extended outflow, with an estimated dynamical age of 3000 yr (André et al. 1990).
Towards VLA 1623 (Fig. 4), the SiO emission is very weak (at its peak at offset $\left(36^{\prime \prime}, 36^{\prime \prime}\right.$ ) it is only $\sim 5 \sigma$ ), and is confined to a small region close to the starless core GPJ2008 Source 4 (Gurney et al. 2008) (we believe the peak in SiO integrated intensity towards the offset $\left(10,-100^{\prime \prime}\right)$ is due to high noise at the map edge). $\mathrm{SiO} 2 \rightarrow 1$ was not detected by $\mathrm{Yu} \&$ Chernin (1997) towards this source, although Garay et al. (2002) detected weak emission towards a similar position as we have found. Note that this position does not lie along the main VLA 1623 outflow axis, which runs in an approximately south-east to north-west direction (Dent et al. 1995). ${ }^{12} \mathrm{CO}$ observations of the GPL2008 source reveal an outflow, although it is possible that this outflow actually emanates from the VLA1623 protostar (Gurney et al. 2008).

The map of the $\mathrm{H}^{13} \mathrm{CO}^{+} 4 \rightarrow 3 / 1 \rightarrow 0$ ratio is complicated because the $\mathrm{H}^{13} \mathrm{CO}^{+} 4 \rightarrow 3$ emission is quite extended, unlike for the other outflows where the emission is confined mainly to the areas around the protostars. The $\mathrm{H}^{13} \mathrm{CO}^{+} 4 \rightarrow 3 / 1 \rightarrow 0$ ratio has two main peaks towards the offsets $\sim\left(50^{\prime \prime}, 80^{\prime \prime}\right)$ and $\sim\left(-10^{\prime \prime},-15^{\prime \prime}\right)$. We select the offset $\left(30^{\prime \prime}, 71^{\prime \prime}\right)$, which is close to one of the peaks in narrow $\mathrm{SiO}$ emission, and the $\mathrm{H}^{13} \mathrm{CO}^{+} 4 \rightarrow 3 / 1 \rightarrow 0$ ratio is also close to its peak here. Furthermore, around this offset, we identified a component at $\sim 2.6 \mathrm{~km} \mathrm{~s}^{-1}$ (component $\mathrm{C} 1$ in Table 3) which is seen in $\mathrm{H}^{13} \mathrm{CO}^{+}$, but is not seen at all in $\mathrm{HN}^{13} \mathrm{C} 1 \rightarrow 0$ (see Fig. 6 and Table 2). Table 3 shows that the excitation of $\mathrm{H}^{13} \mathrm{CO}^{+}$in this component is higher than in the other component at $\sim 3.5 \mathrm{~km} \mathrm{~s}^{-1}$ (C2) which is seen in both molecules. Note also that towards this source, the $\mathrm{HN}^{13} \mathrm{C}$ peaks about $50^{\prime \prime}$ north of the $\mathrm{H}^{13} \mathrm{CO}^{+}$, which could be a chemical effect, but could also be indicative of different conditions between the ion and neutral fluids.

Since the $\mathrm{SiO} 2 \rightarrow 1$ emission is very weak, the spectrum has been smoothed to a velocity resolution $0.5 \mathrm{~km} \mathrm{~s}^{-1}$, and therefore we cannot ascertain its velocity to enough accuracy to be able to tell if it lies at the velocity of $\mathrm{C} 1$ or $\mathrm{C} 2$. $\mathrm{SiO} 8 \rightarrow 7$ was not observed in this outflow due to time constraints.

\subsection{IRAS 20353}

IRAS 20353 is a class I protostar located within the L1152 isolated core (Bontemps et al. 1996), at a distance of 440 pc. It has a bolometric luminosity of $3.3 L_{\odot}$ (Myers et al. 1992), and an ambient velocity of $\sim 3 \mathrm{~km} \mathrm{~s}^{-1}$. Bontemps et al. (1996) detected a bipolar $\mathrm{CO}$ outflow towards this source, which we estimate to have a dynamical age of $65000 \mathrm{yr}^{2}$. This age estimate is rather large in comparison to the other outflows in this study, but note that $65000 \mathrm{yr}$ is the age estimate of the shock at the outer boundary of the outflow lobes, so it is possible that there are younger shocks within the outflow lobes.

Towards IRAS 20353 (Fig. 5), no $\mathrm{SiO} 2 \rightarrow 1$ emission was detected. The $\mathrm{H}^{13} \mathrm{CO}^{+} 4 \rightarrow 3$ emission and the $\mathrm{H}^{13} \mathrm{CO}^{+}$ $4 \rightarrow 3 / 1 \rightarrow 0$ ratio peak towards the central position of the protostar, so we studied the spectra from offset $(0,0)$ in more detail. This way we can investigate if excitation differences between ions and neutrals can be found even in the absence of narrow $\mathrm{SiO}$.

\footnotetext{
${ }^{2}$ Bontemps et al. (1996) give a velocity range of $4-7.5 \mathrm{~km} \mathrm{~s}^{-1}$ for ${ }^{12} \mathrm{CO}$ $(2 \rightarrow 1)$ in the redshifted outflow lobe detected in L1152. Subtracting the ambient source velocity of $\sim 3 \mathrm{~km} \mathrm{~s}^{-1}$ gives a maximum velocity of $\sim 4.5 \mathrm{~km} \mathrm{~s}^{-1}$. The red outflow lobe extends to an offset of approximately $\left(-100^{\prime \prime},-100^{\prime \prime}\right)$, which at the distance of the L1152 cloud, $440 \mathrm{pc}$, corresponds to approximately $0.30 \mathrm{pc}$. This gives a dynamical age estimate for the outflow of $\sim 65000 \mathrm{yr}$.
} 
Towards this offset, we identified two components of emission at $2.6(\mathrm{C} 1)$ and $3.1(\mathrm{C} 2) \mathrm{km} \mathrm{s}^{-1}$. Only the $\mathrm{C} 1$ component was detected in $\mathrm{H}^{13} \mathrm{CO}^{+} 4 \rightarrow 3$ (the peak at $\mathrm{C} 2$ looks like it is a noise spike), although the $\mathrm{H}^{13} \mathrm{CO}^{+} 4 \rightarrow 3 / 1 \rightarrow 0$ integrated intensity ratio in this component is actually lower than the upper limit of the $\mathrm{HN}^{13} \mathrm{C}$ integrated intensity ratio (see Table 3 ). There is therefore no evidence of an over-excitation of $\mathrm{H}^{13} \mathrm{CO}^{+}$.

\section{LVG modelling}

Table 3 shows that towards both NGC 1333 and L1448-IRS3, there is one component of emission which has significantly larger (by $\geq 5-10$ times) $4 \rightarrow 3 / 1 \rightarrow 0$ integrated intensity ratios for $\mathrm{H}^{13} \mathrm{CO}^{+}$compared to $\mathrm{HN}^{13} \mathrm{C}$. Following the analysis of JS06, in Sect. 5.2 we first quantify the $\mathrm{H}^{13} \mathrm{CO}^{+}$and $\mathrm{HN}^{13} \mathrm{C}$ excitation differences by estimating the $\mathrm{H}_{2}$ densities required to explain the line intensity ratios of Table 3, using the large velocity gradient (LVG) approximation. We assume collisional excitation by $\mathrm{H}_{2}$ only, and kinetic temperatures estimated from previous $\mathrm{NH}_{3}$ observations found in the literature. This is to ascertain whether there really is an over-excitation of $\mathrm{H}^{13} \mathrm{CO}^{+}$under these conditions, or if it is just an effect of its lower critical density. In Sect. 5.3, we then investigate the possibility that an electron fractional abundance enhancement could be responsible for the difference in excitation between the two molecules towards NGC 1333 and L1447-IRS3, and we also explore the effect on the excitation of $\mathrm{H}^{13} \mathrm{CO}^{+}$and $\mathrm{HN}^{13} \mathrm{C}$ of varying the gas temperature.

For all of the LVG modelling, we fit the peak intensities of the lines, and following the results given in Table 2 we adopt a line width of $0.8 \mathrm{~km} \mathrm{~s}^{-1}$ for all components. The $\mathrm{H}_{2}$ collisional coefficients used in the LVG model were taken from the Leiden Atomic and Molecular Database (Schöier et al. 2005), which uses the values from Flower (1999) for $\mathrm{H}^{13} \mathrm{CO}^{+}$and Dumouchel et al. (2010) for $\mathrm{HN}^{13} \mathrm{C}$. The electron collisional coefficients were from Faure et al. (priv. comm.) for $\mathrm{H}^{13} \mathrm{CO}^{+}$and Faure et al. (2007) for $\mathrm{HN}^{13} \mathrm{C}$.

\subsection{A note about temperature}

C-shock models predict that the ion and neutral fluid kinetic temperature can increase by several orders of magnitude throughout the propagation of a shock. However, since Jiménez-Serra et al. (2009) found that the narrow $\mathrm{SiO}$ line profile at ambient velocities towards L1448-mm can only be reproduced at very young shock ages $(\sim 3 \mathrm{yr})$, here we only consider very early stages of the precursor where both the ion and neutral fluids have moderate temperatures $(\$ 150 \mathrm{~K})$.

A further complication is that in the magnetic precursor, the neutral, ion and electron fluids are all predicted to have different kinetic temperatures: the ion fluid temperature can be as much as 100 times greater than the neutral temperature, and the electron fluid temperature is predicted to be in-between the ion and neutral temperatures (see e.g. Flower \& Pineau des Forêts 2003). It is also possible that the ion-neutral drift in the precursor could increase the effective temperature of the ion-neutral collisions, similar to the mechanism described in Federman et al. (1996). Strictly speaking, the effective temperature, $T_{\text {eff }}$, that should be used in the LVG model for ion-neutral collisions is given by

$\frac{3}{2} k T_{\mathrm{eff}}=\frac{3}{2} k \frac{m_{\mathrm{i}} T_{\mathrm{n}}+m_{\mathrm{n}} T_{\mathrm{i}}}{m_{\mathrm{i}}+m_{\mathrm{n}}}+\frac{1}{2} \mu v_{\mathrm{in}}^{2}$,

where $m_{\mathrm{n}}\left(m_{\mathrm{i}}\right)$ is the mass of the neutral (ion) species, $T_{\mathrm{n}}\left(T_{\mathrm{i}}\right)$ is the temperature of the neutral (ion) fluid, $\mu$ is the reduced mass of the reacting molecules, $v_{\text {in }}$ is the ion-neutral drift velocity, and $k$ is the Boltzmann constant. The final term of Eq. (1) is the contribution to $T_{\text {eff }}$ from the ion-neutral drift, and this is discussed in more detail in Sect. 5.3.1.

Considering $\mathrm{H}_{2}-\mathrm{H}^{13} \mathrm{CO}^{+}$collisions, when $T_{\mathrm{i}} \gg T_{\mathrm{n}}$, the first term on the right hand side of Eq. (1) tends to $(3 / 2) k\left(m_{\mathrm{H}_{2}} / m_{\mathrm{H}^{13} \mathrm{CO}^{+}}\right) T_{\mathrm{i}}$ (with $\left.m_{\mathrm{H}^{13} \mathrm{CO}^{+}} \sim 15 m_{\mathrm{H}_{2}}\right)$. However, as mentioned above, here we only consider the early stages of the precursor when the ion fluid has not reached such high values. Under these conditions $T_{\mathrm{n}}$ is a good approximation for $T_{\text {eff }}$ (for $T_{\mathrm{i}}<10 T_{\mathrm{n}}$, the $\left.\left[\left(m_{\mathrm{i}} T_{\mathrm{n}}+m_{\mathrm{n}} T_{\mathrm{i}}\right) / m_{\mathrm{i}}+m_{\mathrm{n}}\right)\right]$ term in Eq. (1) approximates to $T_{\mathrm{n}}$ to within $60 \%$ ).

We also use the neutral fluid temperature when considering collisions between electrons and $\mathrm{H}^{13} \mathrm{CO}^{+}$and $\mathrm{HN}^{13} \mathrm{C}$, although strictly speaking the electron temperature should be used. It is currently not possible in our LVG model to use a different kinetic temperature for electrons and for $\mathrm{H}_{2}$, but it is likely that including "hot" electrons would lead to higher $4 \rightarrow 3 / 1 \rightarrow 0$ ratios in both molecules (and hence lower derived electron abundances). Furthermore, since the difference in electron collisional coefficients with $\mathrm{H}^{13} \mathrm{CO}^{+}$and $\mathrm{HN}^{13} \mathrm{C}$ actually decreases for temperatures $\gtrsim 30 \mathrm{~K}$, the inclusion of hot electrons would probably decrease the excitation differences between $\mathrm{H}^{13} \mathrm{CO}^{+}$and $\mathrm{HN}^{13} \mathrm{C}$.

\subsection{Quantifying the $\mathrm{H}^{13} \mathrm{CO}^{+}$and $\mathrm{HN}^{13} \mathrm{C}$ excitation difference}

\subsubsection{NGC 1333 and L1448-IRS3}

For these outflows the differences in integrated intensity ratios between ion and neutral species are similar to those found in L1448-mm, towards the positions where the precursor is believed to have been detected (see JS06, Tables 1 and 2). Since we expect the emission from these species to be optically thin, as was found to be the case for L1448- $\mathrm{mm}$ if the emission is extended (Roberts et al. 2010), the line intensity ratio between different transitions is directly related to the excitation temperature of the gas.

Here we estimate the $\mathrm{H}_{2}$ densities required to explain the line intensity ratios of Table 3 , assuming collisional excitation by $\mathrm{H}_{2}$ only, and kinetic temperatures of $12 \mathrm{~K}$ for both NGC 1333 and L1448-IRS3. The kinetic temperature was estimated by taking the mean kinetic temperature of the $\mathrm{NH}_{3}$ cores identified by Rosolowsky et al. (2008) which lay within $\sim 100^{\prime \prime}$ of the chosen offsets. The estimated $\mathrm{H}_{2}$ densities and column densities are given in Table 4.

We find that for component $\mathrm{C} 1$ of NGC 1333, which has a $4 \rightarrow 3 / 1 \rightarrow 0$ integrated intensity ratio for $\mathrm{H}^{13} \mathrm{CO}^{+} \geq 5$ times greater than that for $\mathrm{HN}^{13} \mathrm{C}$, the required $\mathrm{H}_{2}$ density to excite the $\mathrm{H}^{13} \mathrm{CO}^{+}$is only $\sim 2$ times higher than the upper limit necessary to account for the $\mathrm{HN}^{13} \mathrm{C}$ excitation. This is in contrast to the results found by JS06 for L1448-mm, where the required $\mathrm{H}_{2}$ density for the precursor component was $\gtrsim 10$ times greater for $\mathrm{H}^{13} \mathrm{CO}^{+}$compared to $\mathrm{HN}^{13} \mathrm{C}$, even though the difference in $4 \rightarrow 3 / 1 \rightarrow 0$ ratio between the two molecules was similar to that which we have found. We find a similar result for component C2 of L1448-IRS3, where despite the large difference in integrated intensity ratios between $\mathrm{H}^{13} \mathrm{CO}^{+}$and $\mathrm{HN}^{13} \mathrm{C}$, the $\mathrm{H}_{2}$ density required for $\mathrm{H}^{13} \mathrm{CO}^{+}$is only $\gtrsim 1.5$ times greater than that for $\mathrm{HN}^{13} \mathrm{C}$. For the other components in these outflows, the upper limit of the $\mathrm{H}_{2}$ densities required for $\mathrm{HN}^{13} \mathrm{C}$ are consistent with the $\mathrm{H}_{2}$ density derived for $\mathrm{H}^{13} \mathrm{CO}^{+}$.

A possible reason for the small difference in $\mathrm{H}_{2}$ densities for $\mathrm{H}^{13} \mathrm{CO}^{+}$and $\mathrm{HN}^{13} \mathrm{C}$, despite the large difference in observed integrated intensity ratios, is that the critical density of $\mathrm{HN}^{13} \mathrm{C}$ is 
J. F. Roberts et al.: The search for the magnetic precursor of C-shocks

Table 4. Derived $\mathrm{H}_{2}$ densities and column densities for $\mathrm{H}^{13} \mathrm{CO}^{+}$and $\mathrm{HN}^{13} \mathrm{C}$ for each component observed towards the outflows.

\begin{tabular}{lccccccc}
\hline \hline \multirow{2}{*}{ Outflow } & \multicolumn{2}{c}{$\mathrm{C} 1$} & \multicolumn{2}{c}{$\mathrm{C} 2$} & \multicolumn{2}{c}{$\mathrm{C} 3$} \\
& Molecule & $n\left(\mathrm{H}_{2}\right)\left(\mathrm{cm}^{-3}\right)$ & $N\left(\mathrm{~cm}^{-2}\right)$ & $n\left(\mathrm{H}_{2}\right)\left(\mathrm{cm}^{-3}\right)$ & $N\left(\mathrm{~cm}^{-2}\right)$ & $n\left(\mathrm{H}_{2}\right)\left(\mathrm{cm}^{-3}\right)$ & $N\left(\mathrm{~cm}^{-2}\right)$ \\
\hline NGC 1333 & $\mathrm{H}^{13} \mathrm{CO}^{+}$ & $8.3 \times 10^{6}$ & $8.8 \times 10^{11}$ & $5.8 \times 10^{5}$ & $9.3 \times 10^{11}$ & & \\
& $\mathrm{HN}^{13} \mathrm{C}$ & $\leq 4.4 \times 10^{6}$ & $5.1 \times 10^{11}$ & $\leq 2.9 \times 10^{6}$ & $7.9 \times 10^{11}$ & & \\
L1448-IRS3 & $\mathrm{H}^{13} \mathrm{CO}^{+}$ & $1.5 \times 10^{6}$ & $9.5 \times 10^{11}$ & $1.7 \times 10^{6}$ & $2.4 \times 10^{12}$ & $3.1 \times 10^{6}$ & $8.2 \times 10^{11}$ \\
& $\mathrm{HN}^{13} \mathrm{C}$ & $\leq 6.7 \times 10^{6}$ & $3.5 \times 10^{11}$ & $\leq 1.2 \times 10^{6}$ & $1.9 \times 10^{12}$ & $\leq 5.3 \times 10^{6}$ & $4.3 \times 10^{11}$ \\
VLA 1623 & $\mathrm{H}^{13} \mathrm{CO}^{+}$ & $1.0 \times 10^{6}$ & $6.2 \times 10^{11}$ & $5.9 \times 10^{5}$ & $2.8 \times 10^{12}$ & & \\
& $\mathrm{HN}^{13} \mathrm{C}$ & $\ldots$ & $\ldots$ & $\leq 3.7 \times 10^{6}$ & $9.6 \times 10^{11}$ & & \\
IRAS 20353 & $\mathrm{H}^{13} \mathrm{CO}^{+}$ & $9.4 \times 10^{5}$ & $7.3 \times 10^{11}$ & $\leq 1.1 \times 10^{7}$ & $9.1 \times 10^{10}$ & & \\
& $\mathrm{HN}^{13} \mathrm{C}$ & $\leq 5.1 \times 10^{6}$ & $7.3 \times 10^{11}$ & $\leq 1.5 \times 10^{8}$ & $2.3 \times 10^{11}$ & & \\
& & & & & & & \\
\hline
\end{tabular}

somewhat higher than that for $\mathrm{H}^{13} \mathrm{CO}^{+}$. According to the data given in the LAMDA database (Schöier et al. 2005), at 10-20 K, the critical density of the $\mathrm{H}^{13} \mathrm{CO}^{+} 4 \rightarrow 3$ line is $\sim 8.2 \times 10^{6} \mathrm{~cm}^{-3}$, whereas for same transition of $\mathrm{HN}^{13} \mathrm{C}$ it is more than double, at $\sim 2.0 \times 10^{7} \mathrm{~cm}^{-3}$. A factor of two difference may seem unimportant, but it has a large effect on the densities required to excite the $4 \rightarrow 3$ lines of these molecules. As an example, assuming both molecules have a column density of $1 \times 10^{12} \mathrm{~cm}^{-2}$, a line width of $0.8 \mathrm{~km} \mathrm{~s}^{-1}$, and kinetic temperature of $12 \mathrm{~K}$, the $\mathrm{H}_{2}$ density required to excite $\mathrm{H}^{13} \mathrm{CO}^{+}$to an intensity of $0.2 \mathrm{~K}$ is $\sim 1.2 \times 10^{6} \mathrm{~cm}^{-3}$, whereas for $\mathrm{HN}^{13} \mathrm{C}$ the required density is $\sim 1.2 \times 10^{7} \mathrm{~cm}^{-3}$, an order of magnitude greater. For the analysis of L1448-mm, where large differences in density required to excite the two molecules were found, JS06 derived the $\mathrm{H}_{2}$ densities using the $4 \rightarrow 3 / 3 \rightarrow 2$ ratio for $\mathrm{H}^{13} \mathrm{CO}^{+}$, but since $\mathrm{HN}^{13} \mathrm{C} 4 \rightarrow 3$ and $3 \rightarrow 2$ lines were not detected in the precursor component, they used the upper limit of the $3 \rightarrow 2 / 1 \rightarrow 0$ ratio to derive the $\mathrm{HN}^{13} \mathrm{C}$ densities. If we recalculate the $\mathrm{H}_{2}$ densities of JS06 using the $4 \rightarrow 3 / 1 \rightarrow 0$ ratio in both lines ${ }^{3}$, for the $\left(0,-10^{\prime \prime}\right)$ offset we find that the $\mathrm{H}_{2}$ density required for $\mathrm{H}^{13} \mathrm{CO}^{+}$ is $\sim 2.7 \times 10^{5} \mathrm{~cm}^{-3}$, and for $\mathrm{HN}^{13} \mathrm{C}$ it is $\sim 4.9 \times 10^{5} \mathrm{~cm}^{-3}$. This shows that using this choice of transitions tends to give more similar densities for the two molecules, as expected from the $1 \rightarrow 0$ transition which mainly probes the outer envelope of the cloud. In contrast, the $3 \rightarrow 2$ and $4 \rightarrow 3$ transitions trace higherdensity gas which is likely to be located closer to the regions affected by the outflows and the interaction of the precursor of C-shocks.

Nevertheless, by considering the $1 \sigma$ error in the $\mathrm{H}^{13} \mathrm{CO}^{+}$intensities for component $\mathrm{C} 1$ of NGC 1333 and component $\mathrm{C} 2$ of L1448-IRS3, the lower limits to the $\mathrm{H}_{2}$ densities required for $\mathrm{H}^{13} \mathrm{CO}^{+}\left(6.6 \times 10^{6} \mathrm{~cm}^{-3}\right.$ for $\mathrm{C} 1$ of NGC 1333 and $1.6 \times 10^{6} \mathrm{~cm}^{-3}$ for $\mathrm{C} 2$ of L1448-IRS3) are still higher than the upper limit for the densities derived from $\mathrm{HN}^{13} \mathrm{C}$, therefore there is still evidence to say that, for a gas temperature of $12 \mathrm{~K}$, a single density cannot explain the excitation of both $\mathrm{H}^{13} \mathrm{CO}^{+}$and $\mathrm{HN}^{13} \mathrm{C}$ for these components towards these outflows.

\subsubsection{VLA 1623 and IRAS 20353}

In Table 4 we have also included the estimated $\mathrm{H}_{2}$ densities and column densities for VLA 1623 and IRAS 20353, assuming kinetic temperatures of $17 \mathrm{~K}$ (Wootten et al. 1994) and $10 \mathrm{~K}$ (Benson 1991) for these outflows, respectively. For VLA 1623,

\footnotetext{
3 For this calculation we have converted to main beam temperatures and corrected for beam dilution by multiplying the $4 \rightarrow 3$ intensities by a factor of $\theta_{4 \rightarrow 3}^{2} / \theta_{1 \rightarrow 0}^{2}$, where $\theta_{\mathrm{J}_{\mathrm{up}}-\mathrm{J}_{\mathrm{low}}}$ is the beam size for the transition $J_{\text {up }}-J_{\text {low }}$. For the observations presented in this paper, however, the beam size correction was performed by averaging the spectra of the maps assuming a Gaussian taper of $29^{\prime \prime}$ for all lines.
}

since $\mathrm{HN}^{13} \mathrm{C}$ is not detected in $\mathrm{C} 1$, the component that could be associated with the precursor/shock, we cannot compare the excitation conditions between the two molecules. We do note, however, that the $\mathrm{H}_{2}$ density derived from $\mathrm{H}^{13} \mathrm{CO}^{+}$is higher in $\mathrm{C} 1$ compared to $\mathrm{C} 2$, reflecting the higher excitation in component $\mathrm{C} 1$, which could possibly be associated with shocks or the precursor of shocks.

In IRAS 20353, as expected from the integrated intensity ratios, the densities needed to excite the $\mathrm{H}^{13} \mathrm{CO}^{+}$fall within the upper limits of the densities required for $\mathrm{HN}^{13} \mathrm{C}$, and there is no evidence of any over-excitation in either component.

\subsection{The origin of the apparent $\mathrm{H}^{13} \mathrm{CO}^{+}$over-excitation towards NGC 1333 and L1448-IRS3}

As in JS06, we investigate the possibility that an electron fractional abundance enhancement in these components could be responsible for the difference in excitation between the two molecules, and we also explore the effect of increasing the gas temperature. We first investigate NGC 1333, taking the $\mathrm{H}_{2}$ density derived from $\mathrm{H}^{13} \mathrm{CO}^{+}$in the $\mathrm{C} 2$ component (since it is lower than the upper limits inferred from $\mathrm{HN}^{13} \mathrm{C}$ ), which was $\sim 6 \times 10^{5} \mathrm{~cm}^{-3}$.

Figure 7 compares the effect of increasing the electron fractional abundance (with the temperature fixed at $12 \mathrm{~K}$ ) and increasing the neutral gas temperature (with the electron fractional abundance fixed at $10^{-10}$ ) for both molecules on the $4 \rightarrow 3 / 1 \rightarrow 0$ peak intensity ratio (keeping the column densities constant at $N\left(\mathrm{H}^{13} \mathrm{CO}^{+}\right)=8.8 \times 10^{11} \mathrm{~cm}^{-2}$ and $N\left(\mathrm{HN}^{13} \mathrm{C}\right)=$ $5.1 \times 10^{11} \mathrm{~cm}^{-2}$, the values given in Table 4 for component $\left.\mathrm{C} 1\right)$. It shows that by increasing the electron fractional abundance, the $4 \rightarrow 3 / 1 \rightarrow 0$ ratio of $\mathrm{H}^{13} \mathrm{CO}^{+}$increases rapidly for electron abundances greater than a few times $10^{-5}$. Counterintuitively, the $4 \rightarrow 3 / 1 \rightarrow 0$ ratio of $\mathrm{HN}^{13} \mathrm{C}$ behaves similarly, even though the collisional coefficients for electrons with $\mathrm{H}^{13} \mathrm{CO}^{+}$are $>4$ times greater than those with $\mathrm{HN}^{13} \mathrm{C}$ for these transitions at $12 \mathrm{~K}$. The strong effect of electrons on $\mathrm{HN}^{13} \mathrm{C}$ could be explained by examining the collisional coefficients for each molecule with $\mathrm{H}_{2}$ and electrons: at $12 \mathrm{~K}$, for the $4 \rightarrow 3$ transition of $\mathrm{H}^{13} \mathrm{CO}^{+}$the collision rate with $\mathrm{H}_{2}$ is $4.0 \times 10^{-10} n\left(\mathrm{H}_{2}\right) \mathrm{s}^{-1}$, and the collision rate with electrons is $1.4 \times 10^{-5} n\left(\mathrm{H}_{2}\right) \mathrm{X}(\mathrm{e}) \mathrm{s}^{-1}$, meaning that the critical electron fractional abundance (which we define as the value for which the collisional rates for $\mathrm{H}_{2}$ and electrons are equal) is $\sim 3 \times 10^{-5}$. For $\mathrm{HN}^{13} \mathrm{C}$ the collisional rates are $1.1 \times 10^{-10} n\left(\mathrm{H}_{2}\right) \mathrm{s}^{-1}$ and $3.1 \times 10^{-6} n\left(\mathrm{H}_{2}\right) \mathrm{X}(\mathrm{e}) \mathrm{s}^{-1}$ with $\mathrm{H}_{2}$ and electrons respectively, meaning that the critical electron fractional abundance is also a few times $10^{-5}$. Consequently, electrons begin to affect both molecules once the electron abundance is $\gtrsim 10^{-5}$.

We find that a fractional electron abundance of $8.8 \times 10^{-4}$ is needed to reproduce the observed $\mathrm{H}^{13} \mathrm{CO}^{+} 4 \rightarrow 3 / 1 \rightarrow 0$ ratio 


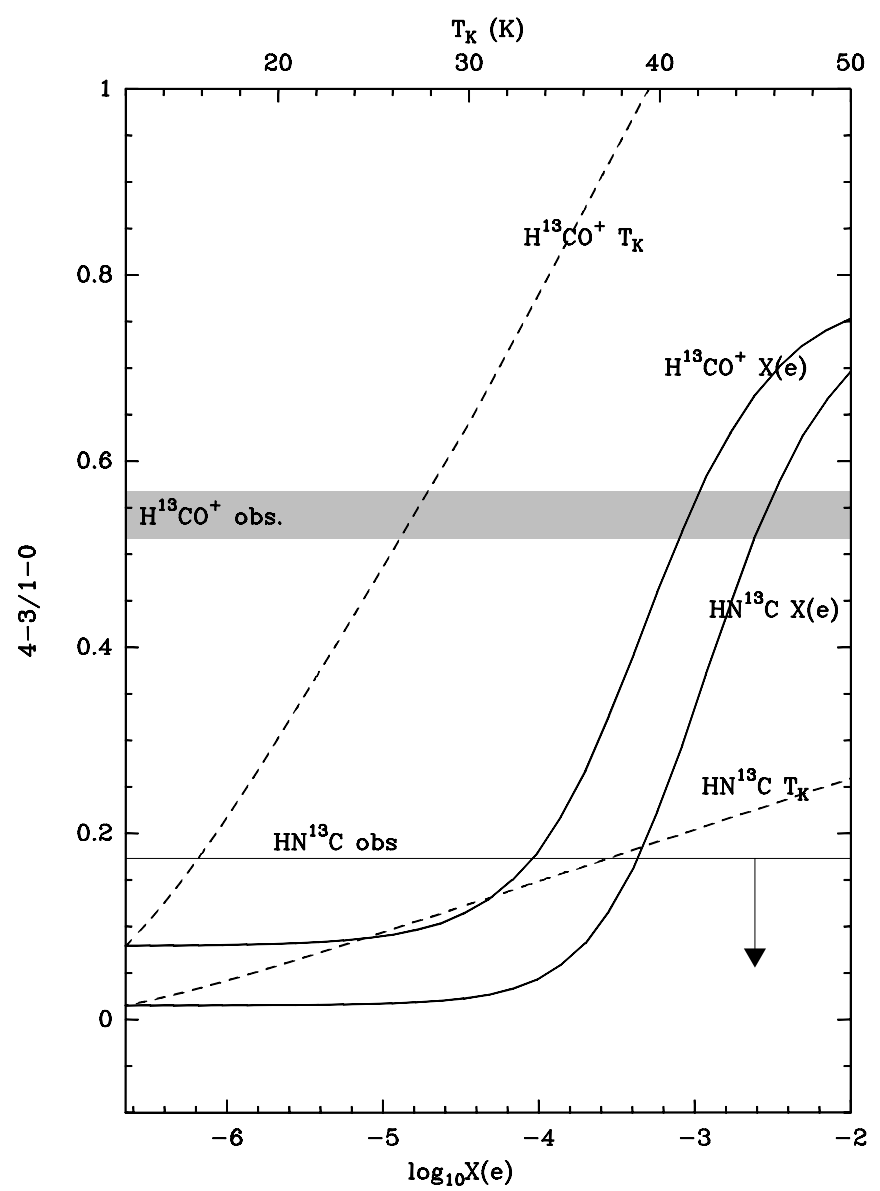

Fig. 7. Plot showing the effect of increasing electron fractional abundance (thick black curves, calculated using a constant kinetic temperature of $12 \mathrm{~K}$ ) and kinetic temperature (dashed curves, calculated using a negligible electron abundance) on the $4 \rightarrow 3 / 1 \rightarrow 0$ line intensity ratios of $\mathrm{H}^{13} \mathrm{CO}^{+}$and $\mathrm{HN}^{13} \mathrm{C}$, for gas with an $\mathrm{H}_{2}$ density of $6 \times$ $10^{5} \mathrm{~cm}^{-3}$ and $\mathrm{H}^{13} \mathrm{CO}^{+}$and $\mathrm{HN}^{13} \mathrm{C}$ column densities of $8.8 \times 10^{11} \mathrm{~cm}^{-2}$ and $5.1 \times 10^{11} \mathrm{~cm}^{-2}$ respectively. The grey bar shows the observed $\mathrm{H}^{13} \mathrm{CO}^{+} 4 \rightarrow 3 / 1 \rightarrow 0$ line intensity ratio towards NGC 1333 , taking into account the $1 \sigma$ errors in the observations. The thin black line shows the upper limit to the observed $\mathrm{HN}^{13} \mathrm{C} 4 \rightarrow 3 / 1 \rightarrow 0$ line intensity ratio towards NGC 1333.

(assuming that the electron temperature is equal to the neutral temperature of $12 \mathrm{~K}$ ). However, for this electron abundance the $\mathrm{HN}^{13} \mathrm{C} 4 \rightarrow 3 / 1 \rightarrow 0$ is 0.31 , almost double the observed upper limit. In addition, this electron fractional abundance exceeds the value of $\leqslant 10^{-5}$ predicted by C-shock models (Flower et al. 1996; Flower \& Pineau des Forêts 2003). As pointed out by JS06, the cosmic abundance of atomic carbon $\left(\mathrm{X}(\mathrm{C}) \sim(2-3) \times 10^{-4}\right)$, the main repository of positive charge in dark clouds, provides a limit for the maximum electron abundance, which is also exceeded by this estimate.

On the other hand, Fig. 7 shows that increasing the kinetic temperature is more effective at increasing the $4 \rightarrow 3 / 1 \rightarrow 0$ ratio for $\mathrm{H}^{13} \mathrm{CO}^{+}$compared to $\mathrm{HN}^{13} \mathrm{C}$. This can be explained by considering the critical densities of the molecules as follows: at densities much greater than the critical density, when collisions completely dominate, the excitation temperature of a transition tends towards the kinetic temperature for an optically thin transition (Green 1985), and the $4 \rightarrow 3 / 1 \rightarrow 0$ ratio has a relatively strong temperature dependence, proportional to $\exp \left[-\left(\Delta E_{43}-\Delta E_{10}\right) / k T_{\text {kin }}\right]$, where $\Delta E_{i j}$ is the energy difference between levels $i$ and $j$, and $T_{\text {kin }}$ is the gas kinetic temperature.
At the other extreme, at low densities, radiative excitation dominates and the ratio is independent of the gas kinetic temperature. At a gas density of $6 \times 10^{5} \mathrm{~cm}^{-3}$, the excitation of both molecules is in-between the two regimes, but it appears that since the $\mathrm{H}^{13} \mathrm{CO}^{+}$critical densities are a factor of two lower than for $\mathrm{HN}^{13} \mathrm{C}$, the temperature dependence of $\mathrm{H}^{13} \mathrm{CO}^{+}$is closer to the collisionally dominated regime, and hence stronger temperature dependence, than $\mathrm{HN}^{13} \mathrm{C}$.

From Fig. 7, we see that in order to reproduce the $4 \rightarrow 3 / 1 \rightarrow 0$ ratio of $\mathrm{H}^{13} \mathrm{CO}^{+}$, a temperature of $\sim 27 \mathrm{~K}$ is needed. At this temperature, the $4 \rightarrow 3 / 1 \rightarrow 0$ ratio of $\mathrm{HN}^{13} \mathrm{C}$ is $\sim 0.10$, which is below the observed upper limit of 0.17 . The LVG model shows that the $4 \rightarrow 3 / 1 \rightarrow 0$ ratios of both $\mathrm{H}^{13} \mathrm{CO}^{+}$and $\mathrm{HN}^{13} \mathrm{C}$ are approximately constant with column density for column densities $\lesssim 10^{12} \mathrm{~cm}^{-2}$ (plot not shown), so by adjusting the column densities of $\mathrm{H}^{13} \mathrm{CO}^{+}$and $\mathrm{HN}^{13} \mathrm{C}$ slightly, to $6.9 \times 10^{11} \mathrm{~cm}^{-2}$ and $4.1 \times 10^{11} \mathrm{~cm}^{-2}$ respectively, the absolute intensities of each line can be fitted to within $10 \%$.

Repeating the analysis for L1448-IRS3, assuming a gas density of $1 \times 10^{6} \mathrm{~cm}^{-3}$ (as derived from the upper limit $\mathrm{HN}^{13} \mathrm{C}$ for the $\mathrm{C} 2$ component), we find that a temperature of $14 \mathrm{~K}$ or an electron fractional abundance of $5.4 \times 10^{-5}$ can explain the $\mathrm{H}^{13} \mathrm{CO}^{+} 4 \rightarrow 3 / 1 \rightarrow 0$ ratio. However, for both of these conditions, the $\mathrm{HN}^{13} \mathrm{C} 4 \rightarrow 3 / 1 \rightarrow 0$ ratio is predicted to be slightly above the observed value (the temperature increase predicts a value $24 \%$ higher than the upper limit and the electron fractional abundance increase predicts a value $54 \%$ higher). This is probably because the density of the gas used was derived from $\mathrm{HN}^{13} \mathrm{C}$ upper limit, so of course if the temperature or electron density is increased, it will increase the excitation of $\mathrm{HN}^{13} \mathrm{C}$ so it will no longer fit the observations. In fact, since the density is only an upper limit, one finds that if the density is decreased to $3 \times 10^{5} \mathrm{~cm}^{-3}$, the $\mathrm{H}^{13} \mathrm{CO}^{+}$ratio can be fitted with a temperature of $27 \mathrm{~K}$, and under these conditions the $\mathrm{HN}^{13} \mathrm{C}$ ratio is only over-predicted by $10 \%$. At this density, increasing the electron abundance to $3.7 \times 10^{-4}$ can also fit the $\mathrm{H}^{13} \mathrm{CO}^{+}$ratio, but in this case the $\mathrm{HN}^{13} \mathrm{C}$ ratio is over-predicted by $68 \%$.

For both of these outflows, therefore, it seems that the overexcitation of $\mathrm{H}^{13} \mathrm{CO}^{+}$can be explained best by a single density and a slightly raised temperature in the affected velocity component with respect to the other ones for both $\mathrm{H}^{13} \mathrm{CO}^{+}$and $\mathrm{HN}^{13} \mathrm{C}$. Given that using the same kinetic temperature and density for each species fits the observations reasonably well, we can not conclude that an electron fractional abundance increase is needed to explain the $\mathrm{H}^{13} \mathrm{CO}^{+}$over-excitation. Nor can we unequivocally say that the $\mathrm{H}^{13} \mathrm{CO}^{+}$and $\mathrm{HN}^{13} \mathrm{C}$ have different excitation conditions in the velocity components with the high $4 \rightarrow 3 / 1 \rightarrow 0$ ratio for $\mathrm{H}^{13} \mathrm{CO}^{+}$compared to $\mathrm{HN}^{13} \mathrm{C}$. Therefore we can not confirm that the difference in $4 \rightarrow 3 / 1 \rightarrow 0$ ratios is due to the magnetic precursor.

\subsubsection{Considering the effect of ion-neutral drift}

According to Eq. (1), the effective temperature of the $\mathrm{H}_{2}-\mathrm{H}^{13} \mathrm{CO}^{+}$collisions could be enhanced by a factor of $(1 / 2) \mu v_{\text {in }}^{2}$, due to ion-neutral drift. If this were the case here, we would expect the temperature needed to fit $\mathrm{H}^{13} \mathrm{CO}^{+}$to be greater than that needed to fit $\mathrm{HN}^{13} \mathrm{C}$. For the case of NGC 1333, from Fig. 7 it is clear that $\mathrm{HN}^{13} \mathrm{C}$ can have a temperature of anything $\$ 37 \mathrm{~K}$ for its $4 \rightarrow 3 / 1 \rightarrow 0$ ratio to remain below the observed upper limit. It is therefore possible that $\mathrm{HN}^{13} \mathrm{C}$ temperature could be lower than the $\sim 27 \mathrm{~K}$ needed to fit $\mathrm{H}^{13} \mathrm{CO}^{+}$. Similarly for L1448-IRS3, if the $\mathrm{HN}^{13} \mathrm{C}$ temperature is kept at $12 \mathrm{~K}$ and the 
$\mathrm{H}^{13} \mathrm{CO}^{+}$temperature is increased by only a few degrees $\mathrm{K}$, both lines can be fitted perfectly using the same gas density. However, since C-shock models predict that the ion-neutral drift velocity could be as high as $\sim 30 \mathrm{~km} \mathrm{~s}^{-1}$ (Flower et al. 1996), this would lead to a $T_{\text {eff }}$ for $\mathrm{H}_{2}-\mathrm{H}^{13} \mathrm{CO}^{+}$collisions of $\sim 68000 \mathrm{~K}$. Even at a more modest ion-neutral drift velocity of $5 \mathrm{~km} \mathrm{~s}^{-1}, T_{\text {eff }}$ would be as high as $\sim 1900 \mathrm{~K}$. One would therefore expect the excitation of $\mathrm{H}^{13} \mathrm{CO}^{+}$to be characterised by a much higher effective temperature than the increases of $2-15 \mathrm{~K}$ which we have found.

\section{Discussion and conclusions}

Following previous studies on L1448-mm (e.g. Jiménez-Serra et al. 2004; and JS06), which have interpreted the narrow $\mathrm{SiO}$ emission at ambient velocities and high line intensity ratios of ions compared to neutral species as signatures of the magnetic precursor of C-shocks, we have searched for these features towards four other young, low-mass protostars (class 0/I) with outflows. The sources NGC 1333, L1448-IRS3, and VLA 1623 all show narrow $\mathrm{SiO} 2 \rightarrow 1$ emission, and NGC 1333 and L1448-IRS3 also show velocity components with a much higher $4 \rightarrow 3 / 1 \rightarrow 0$ integrated intensity ratio for $\mathrm{H}^{13} \mathrm{CO}^{+}$compared to $\mathrm{HN}^{13} \mathrm{C}$, similar to that seen towards L1448-mm. VLA 1623 showed a component in $\mathrm{H}^{13} \mathrm{CO}^{+}$which is not seen at all in $\mathrm{HN}^{13} \mathrm{C}$, even in the $1 \rightarrow 0$ line, which could either indicate a large difference in excitation conditions, or in abundances, between the two molecules.

JS06 explained the high $4 \rightarrow 3 / 1 \rightarrow 0$ integrated intensity ratio of $\mathrm{H}^{13} \mathrm{CO}^{+}$by the selective excitation of ions by electrons, which have an enhanced fractional abundance during the magnetic precursor stage of C-shocks. Our LVG analysis has found that for the sources NGC 1333 and L1448-IRS3, an increase in electron fractional abundance would cause the $\mathrm{HN}^{13} \mathrm{C}$ $4 \rightarrow 3 / 1 \rightarrow 0$ intensity ratios to be increased beyond their observed upper limits. Instead we find that using a slightly higher kinetic temperature (an increase of $\lesssim 15 \mathrm{~K}$ ) than the value estimated from previous $\mathrm{NH}_{3}$ observations can fit the intensities of both molecules well. It is also possible to fit the intensities with a higher temperature for $\mathrm{H}^{13} \mathrm{CO}^{+}$than for $\mathrm{HN}^{13} \mathrm{C}$, which would be expected in the magnetic precursor due to the increase in effective temperature of ion-neutral collisions. However, the estimated differences in temperature needed to fit the observations are much smaller than would be expected for the typical ion-neutral drift velocities predicted by $\mathrm{C}$-shock models. Given that the $\mathrm{H}^{13} \mathrm{CO}^{+} 4 \rightarrow 3$ integrated intensity in Figs. 2 and 3 tends to peak towards the protostars, it could simply be that the high $\mathrm{H}^{13} \mathrm{CO}^{+} 4 \rightarrow 3 / 1 \rightarrow 0$ ratio is caused by protostellar heating. However, since the errors involved in measuring the observations are large, we do not rule out the possibility that electrons play a part in the excitation of $\mathrm{H}^{13} \mathrm{CO}^{+}$. In fact, since $\mathrm{C}$-shock models predict both a temperature and electron abundance increase (e.g. Flower et al. 1996), it is possible that a combination of temperature and electron abundance increase are responsible for the high $\mathrm{H}^{13} \mathrm{CO}^{+} 4 \rightarrow 3 / 1 \rightarrow 0$ ratios. High-angular resolution interferometric observations are therefore required to separate the protostar and precursor contributions to the high $4 \rightarrow 3 / 1 \rightarrow 0$ ratios observed towards these outflows.

Lefloch et al. (1998b) and Codella et al. (1999) suggested that the narrow, ambient $\mathrm{SiO}$ detected towards NGC 1333 could have been produced in post-shock gas which has decelerated to ambient velocities, rather than being produced at the magnetic precursor stage. Codella et al. (1999) estimated that for typical outflows this could take of the order $10^{4} \mathrm{yr}$, but the dynamical timescales of the shocks towards L1448-IRS3 and VLA 1623 are much shorter than this. Furthermore, it seems that the $\mathrm{SiO} 2 \rightarrow 1$ emission towards these two outflows is on a much smaller scale than towards NGC 1333, which could indicate that the narrow $\mathrm{SiO}$ is produced by a smaller, local effect. In addition, towards VLA 1623, the narrow SiO lies in a small area off the outflow axis, so it might be difficult to explain how post-shock gas could arrive at that location. It is possible that the $\mathrm{SiO}$ is produced in the post shock gas from larger scale outflows that may exist in the region (Jiménez-Serra et al. 2010). If this is the case, one would not expect the narrow $\mathrm{SiO}$ to be compact and confined to regions near the protostars, as found towards L1448-mm (Jiménez-Serra et al. 2011). Large scale and high sensitivity maps would be useful to determine the absolute extent of the emission.

In summary, we cannot confirm that we have found the magnetic precursor towards these outflows, although the theory that ambient $\mathrm{SiO}$ is produced during the precursor stage of $\mathrm{C}$-shocks remains very plausible. VLA 1623 appears to be a very promising outflow for further study, since the region of $\mathrm{SiO} 2 \rightarrow 1$ emission is small and weak it could be that it is in the early stages of formation, and there is a component of $\mathrm{H}^{13} \mathrm{CO}^{+}$that is not seen in $\mathrm{HN}^{13} \mathrm{C}$. Large scale interferometric maps of the narrow $\mathrm{SiO}$, plus maps of several different ion and neutral species will be essential to provide conclusive detection of this phenomenon.

Acknowledgements. We would like to thank the anonymous referee for helpful comments on an earlier draft of this paper. This paper is based on observations from the IRAM 30 m Telescope and the James Clerk Maxwell Telescope (project M10BU04). The James Clerk Maxwell Telescope is operated by the Joint Astronomy Centre on behalf of the Science and Technology Facilities Council of the United Kingdom, The Netherlands Organisation for Scientific Research, and the National Research Council of Canada. IRAM is supported by INSU/CNRS (France), MPG (Germany) and IGN (Spain). This work has made use of the NASA/ IPAC Infrared Science Archive, which is operated by the Jet Propulsion Laboratory, California Institute of Technology, under contract with the National Aeronautics and Space Administration. This work has been partially supported by the Spanish Ministerio de Ciencia e Innovación under project ESP2007- 65812-C02-01, and by the Comunidad de Madrid Government under PRICIT project S-0505/ESP-0237 (ASTROCAM). A. Gusdorf acknowledges support by the grant ANR-09-BLAN-0231-01 from the French Agence Nationale de la Recherche as part of the SCHISM project.

\section{References}

André, P., Martín-Pintado, J., Despois, D., \& Montmerle, T. 1990, A\&A, 236, 180

Bachiller, R., Martín-Pintado, J., Tafalla, M., Cernicharo, J., \& Lazareff, B. 1990, A\&A, 231, 174

Bachiller, R., Guilloteau, S., Gueth, F., et al. 1998, A\&A, 339, L49

Benson, P. J. 1991, ASPC, 16, 21

Bontemps, S., André, P., Terebey, S., \& Cabrit, S. 1996, A\&A, 311, 858

Caselli, P., Hartquist, T. W., \& Havnes, O. 1997, A\&A, 322, 296

Cernis, K. 1990, Ap\&SS, 166, 315

Choi, M., Kamazaki, T., Tatematsu, K., \& Panis, J.-F. 2004, ApJ, 617, 1157

Ciardi, D. R., Telesco, C. M., Williams, J. P., et al. 2003, ApJ, 585, 392

Codella, C., Bachiller, R., \& Reipurth, B. 1999, A\&A, 343, 585

Dent, W. R. F., Matthews, H. E., \& Walther, D. M. 1995, MNRAS, 277, 193

Draine, B. T. 1980, ApJ, 241, 1021

Dumouchel, F., Faure, A., \& Lique, F. 2010, MNRAS, 406, 2488

Faure, A., Varambhia, H. N., Stoecklin, T., \& Tennyson, J. 2007, MNRAS, 382, 840

Federman, S. R., Rawlings, J. M. C., Taylor, S. D., \& Williams, D. A. 1996, MNRAS, 279, L41

Flower, D. R. 1999, MNRAS, 305, 651

Flower, D. R., \& Pineau des Forêts, G. 2003, MNRAS, 343, 390

Flower, D. R., Pineau des Forêts, G., Field, D., \& May, P. W. 1996, MNRAS, 280,447

Garay, G., Mardones, D., Rodríguez, L. F., Caselli, P., \& Bourke, T. L. 2002, ApJ, 567, 980

Green, S. 1985, Il Nuovo Cimento C, 8, 435

Gurney, M., Plume, R., \& Johnstone, D. 2008, PASP, 120, 873 
Gusdorf, A., Cabrit, S., Flower, D. R., \& Pineau des Forêts, G. 2008, A\&A, 482, 809

Herbig, G. H., \& Jones, B. F. 1983, AJ, 88, 1040

Hirota, T., Yamamoto, S., Mikami, H., \& Ohishi, M. 1998, ApJ, 503, 717

Jiménez-Serra, I., Martín-Pintado, J., Rodríguez-Franco, A., \& Marcelino, N. 2004, ApJ, L49

Jiménez-Serra, I., Martín-Pintado, J., Viti, S., et al. 2006, ApJ, 650, L135 [JS06] Jiménez-Serra, I., Caselli, P., Martín-Pintado, J., \& Hartquist, T. W. 2008, A\&A, 482, 549

Jiménez-Serra, I., Martín-Pintado, J.,Caselli, P., Viti, S., \& Rodríguez-Franco, A. 2009, ApJ, 695, 149

Jiménez-Serra, I., Caselli, P., Tan, J. C., et al. 2010, MNRAS, 406, 187

Jiménez-Serra, I., Martín-Pintado, J., Winters, J.-M., Rodríguez-Franco, A., \& Caselli, P. 2011, ApJ, 739, 80

Langer, W. D., Castets, A., \& Lefloch, B. 1996, ApJ, 471, 111

Lefloch, B., Castets, A., Cernicharo, J., Langer, W. D., \& Zylka, R. 1998a, A\&A, 334, 269
Lefloch, B., Castets, A., Cernicharo, P., \& Loinard, L. 1998b, ApJ, 504, L109 Martín-Pintado, J., Bachiller, R., \& Fuente, A. 1992, A\&A, 254, 315

Mullan, D. J. 1971, MNRAS, 153, 145

Myers, P., Fuller, G., Mathieu, R., et al. 1987, ApJ, 319, 340

Roberts, J. F., Jiménez-Serra, I., Martín-Pintado, J., et al. 2010, A\&A, 513, A64

Rosolowsky, E. W., Pineda, J. E., Foster, J. B., et al. 2008, ApJS, 175, 509

Sandell, G., \& Knee, L. B. G. 2001, ApJ, 546, L49

Sandell, G., Aspin, C., Duncan, W. D., Robson, E. I., \& Dent, W. R. F. 1990 A\&A, 232, 347

Sandell, G., Aspin, C., Duncan, W. D., Russell, A. P. G., \& Robson, E. I. 1991, ApJ, 376, 17

Schöier, F. L., van der Tak, F. F. S., van Dishoeck, E. F., \& Black, J. H. 2005, A\&A, 432369

Woodall, J., Agúndez, M., Markwick-Kemper, A. J., \& Millar, T. J. 2007, A\&A, 466, 1197

Wootten, A., André, P., Despois, D., \& Sargent, A. 1994, ASPC, 65, 294

Yu, T., \& Chernin, L. M. 1997, ApJ, 479, L63 